\title{
Voter Records Interchange Common Data Format Specification
}

John Wack

Sam Dana

John Dziurlaj

E. John Sebes

Paul Stenbjorn

Sarah Whitt

This publication is available free of charge from:

https://doi.org/10.6028/NIST.SP.1500-102

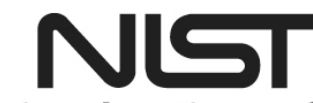

National Institute of Standards and Technology 
NIST Special Publication 1500-102

\title{
Voter Records Interchange Common Data Format Specification
}

Version 1.0

\author{
John Wack \\ Software and Systems Division \\ Information Technology Laboratory, NIST \\ Sam Dana \\ Prometheus Computing \\ John Dziurlaj \\ Democracy Fund \\ E. John Sebes \\ OSET Institute \\ Paul Stenbjorn \\ Democracy Fund \\ Sarah Whitt \\ Electronic Registration Information Center
}

This publication is available free of charge from:

https://doi.org/10.6028/NIST.SP.1500-102

November 2019

INCLUDES UPDATES AS OF 03-31-2020; SEE APPENDIX E

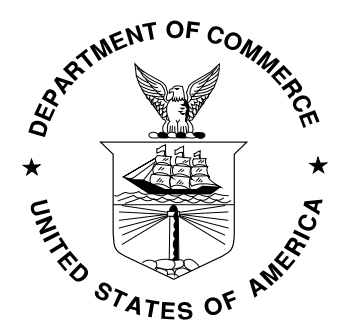

U. S. Department of Commerce Wilbur Ross, Secretary

National Institute of Standards and Technology Walter G. Copan, Under Secretary of Commerce for Standards and Technology and Director 


\title{
National Institute of Standards and Technology (NIST) Special Publication 1500-102
}

\author{
104 pages (March 2020)
}

NIST Special Publication series 1500 is intended to capture external perspectives related to NIST standards, measurement, and testing-related efforts. These external perspectives can come from industry, academia, government, and others. These reports are intended to document external perspectives and do not necessarily represent official NIST positions.

Certain commercial entities, equipment, or materials may be identified in this document in order to describe an experimental procedure or concept adequately. Such identification is not intended to imply recommendation or endorsement by NIST, nor is it intended to imply that the entities, materials, or equipment are necessarily the best available for the purpose.

There may be references in this publication to other publications currently under development by NIST in accordance with its assigned statutory responsibilities. The information in this publication, including concepts and methodologies, may be used by federal agencies even before the completion of such companion publications. Thus, until each publication is completed, current requirements, guidelines, and procedures, where they exist, remain operative. For planning and transition purposes, federal agencies may wish to closely follow the development of these new publications by NIST.

Organizations are encouraged to review all draft publications during public comment periods and provide feedback to NIST. All NIST publications are available at http://www.nist.gov/publication-portal.cfm.

National Institute of Standards and Technology

Attn: Software and Systems Division, Information Technology Laboratory

100 Bureau Drive (Mail Stop 8970) Gaithersburg, MD 20899-8930

Email: voting@nist.gov 


\title{
Reports on Computer Systems Technology
}

The Information Technology Laboratory (ITL) at the National Institute of Standards and Technology (NIST) promotes the U.S. economy and public welfare by providing technical leadership for the Nation's measurement and standards infrastructure. ITL develops tests, test methods, reference data, proof of concept implementations, and technical analyses to advance the development and productive use of information technology. This document reports on ITL's research, guidance, and outreach efforts in Information Technology and its collaborative activities with industry, government, and academic organizations.

\begin{abstract}
This document presents a specification for voter records data interchange related to voter registration, i.e., registration requests from online voter registration made to voter registration systems, and responses to the requests returning from the voter registration system. The specification includes XML (eXtensible Markup Language) and JSON (JavaScript Object Notation) schemas.
\end{abstract}

\section{Keywords}

Common data format (CDF); Federal Post Card Application (FPCA); JavaScript Object Notation (JSON); National Voter Registration Act (NVRA); voter registration; eXtensible Markup Language (XML). 


\section{Acknowledgements}

In the development of this specification, the authors recognize the significant contributions made by individuals and organizations involved in the NIST Voting Interoperability Public Working Group as well as in the public and private sectors, whose thoughtful and constructive comments improved the overall quality, thoroughness, and usefulness of this publication. The authors gratefully acknowledge and appreciate the following contributors for their keen and insightful assistance with developing this specification:

\begin{tabular}{|c|c|c|}
\hline Kim Brace & Thomas Connolly & Monica Crane-Childers \\
\hline Election Data Services, Inc. & NY State Board of Elections & Democracy Works \\
\hline Ericka Haas & Katy Owens Hubler & Christopher Jerdonek \\
\hline ERIC, Inc. & Democracy Research, LLC & $\begin{array}{l}\text { San Francisco Elections } \\
\text { Commission }\end{array}$ \\
\hline $\begin{array}{l}\text { Jared Marcotte } \\
\text { The Turnout }\end{array}$ & Lauren Massa-Lochridge & $\begin{array}{l}\text { John McCarthy } \\
\text { Verified Voting }\end{array}$ \\
\hline Janet Modrow & Tammy Patrick & Jen Tolentino \\
\hline Florida Division of Elections & Democracy Fund & Rock the Vote \\
\hline $\begin{array}{l}\text { David Wallick } \\
\text { Everyone Counts }\end{array}$ & $\begin{array}{l}\text { Stafford Ward } \\
\text { Open Source Election } \\
\text { Technology Institute }\end{array}$ & \\
\hline
\end{tabular}

The editor also wishes to thank his colleagues of the National Institute of Standards and Technology Voter Records Interchange Public Working Group, who contributed to its technical content, including Mary Brady, Gema Howell, and Benjamin Long. 


\section{Executive Summary}

Voter records are exchanged between an increasing number of entities. Whether it is voter registration offered during an interaction at the motor vehicles administration (MVA), an update to a voter record using an online portal, records exchanged as part of a voter matching database, or requests for absentee ballots, all of these systems need a common data format to effectively communicate.

However, the data exchanged is generally in a non-uniform format. This can cause a number of complications in that each state/territory, or sometimes each individual portal application, may have its own format that must be interpreted and translated on the receiving end. Voter addresses, in particular, are often provided to voter registration (VR) authorities in formats that are difficult to efficiently store in voter registration databases and use in various related applications. The collection of accurate voter registration address information is central to the routing of voter registration requests and subsequently in assigning voters to election districts. Each voter records application being developed must then be aware of each state's specific formats or design its own format, which complicates development and inhibits the implementation of digital voter record systems.

This specification assists election officials and developers in more easily implementing and supporting the development of online voter registration (OVR) systems by providing them with a uniform common data format for voter records interchange (VRI), that is, the voter registration requests and responses needed for OVR and for voter records maintenance. The languages used in the common data format are XML (eXtensible Markup Language) and JSON (JavaScript Object Notation).

The advantages of using this specification include:

- Providing a ready data interchange format for OVR systems to remove the need for individual system development projects to define their own data models and formats.

- Assisting election officials by reducing or eliminating non-standard exchange formats for voter registration data. Currently, the systems involved and data they produce do not interoperate, adding complexity to the process.

- Providing a baseline CDF (common data format) for voter registration data that can be continually refined to be more efficient and adaptable across all states. Once jurisdictions adopt the CDF VRI, their experience and feedback will refine the continued development of the specification.

- Providing the foundation for additional use cases in the future, which could include:

- matching driver's license data between the MVA and voter record systems,

- automated notifications between the MVA and voter record systems,

o interoperability with electronic pollbooks,

- voter record maintenance activities,

- cross-state record matching, or 
$\circ$ facilitating data reporting for the Election Administration and Voting Survey (EAVS).

This specification provides background and explanation of how online voter registration typically works, using the data required by the National Voter Registration Act (NVRA) and Federal Post Card Application (FPCA) voter registration forms, including state-specific additions to these forms. It then contains an explanation of a UML (Unified Markup Language) model that was created to detail the data elements required in voter registration requests and responses. The UML model was used to generate XML and JSON schemas, which are both explained and used in various implementation examples.

The intended audience of this specification includes election officials, VR system designers and developers, and others in the election community including the general public. Some background in election administration and registration is useful in understanding the material in this specification. 


\section{Table of Contents}

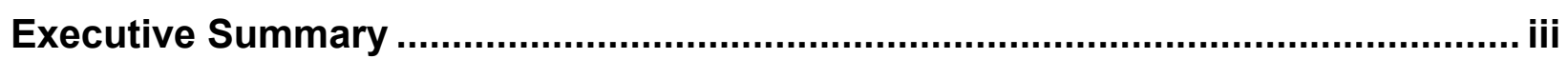

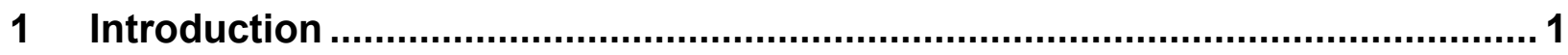

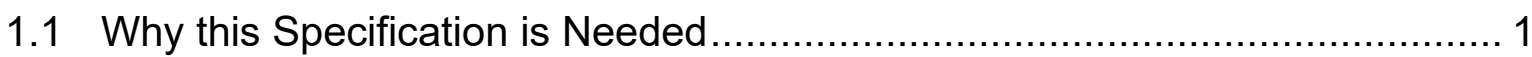

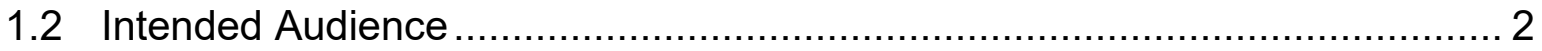

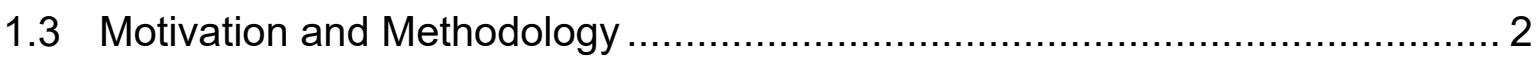

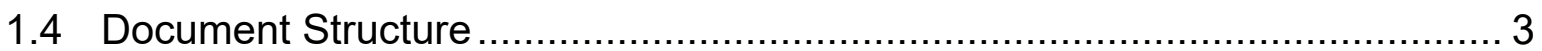

2 Overview of Digital OVR Transactions ……........................................................ 5

2.1 Voter Records Request...................................................................... 7

2.2 Voter Records Response ................................................................. 11

2.3 Including State-specific Request and Response Items .............................. 13

2.4 Dealing with Addresses - The U.S. Thoroughfare, Landmark, and Postal Address Data Standard ....................................................................... 14

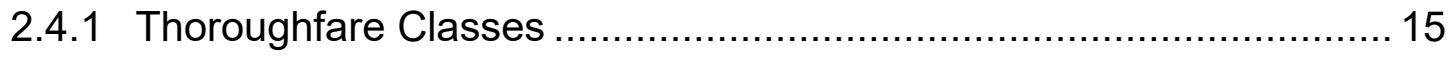

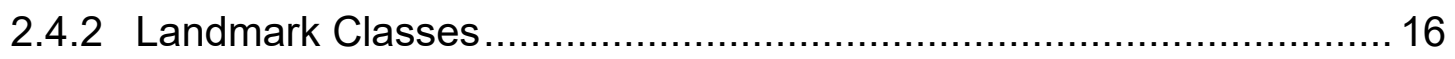

2.4.3 Postal Delivery Classes ............................................................... 17

2.4.4 General Address Class and Handling Non-U.S. Addresses .............. 17

3 Voter Records Interchange UML Model Documentation ................................. 19

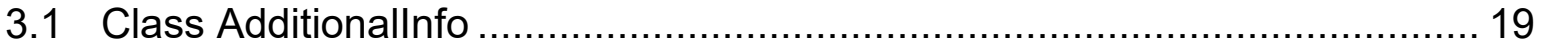

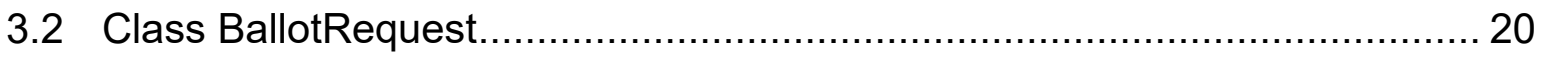

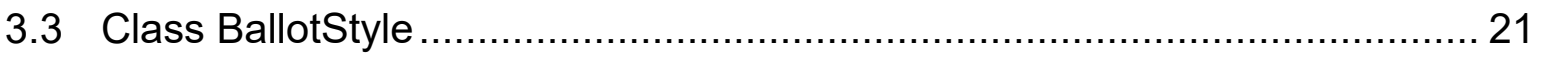

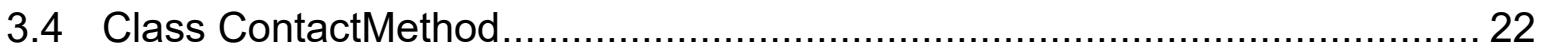

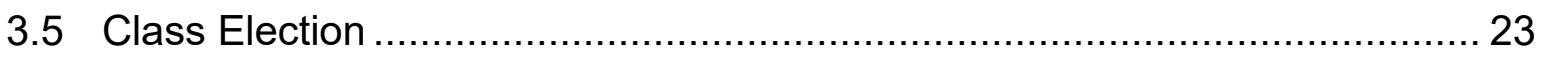

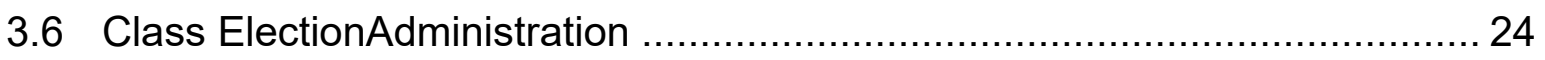

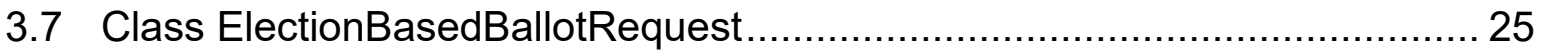

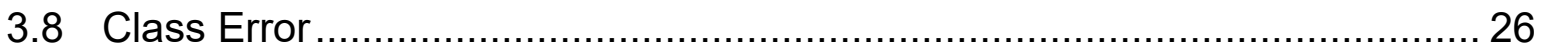

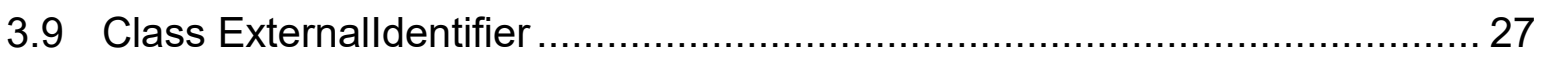

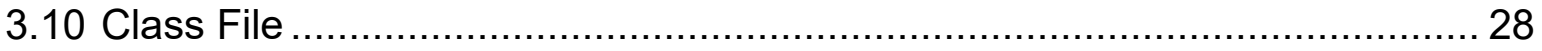

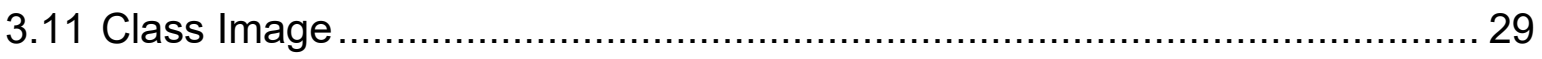

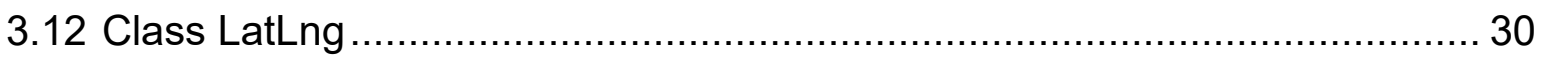

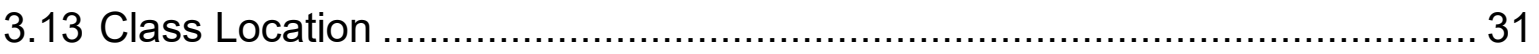




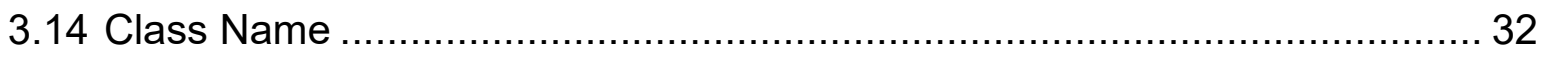

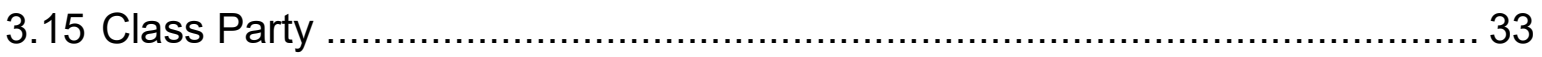

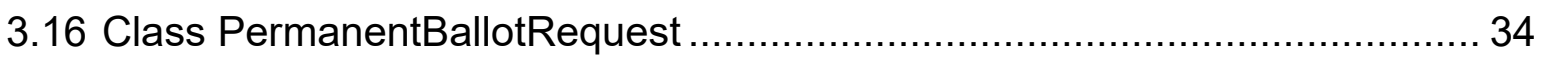

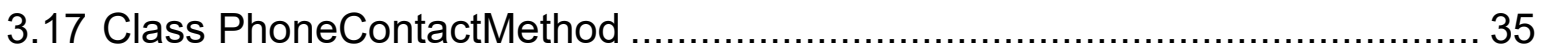

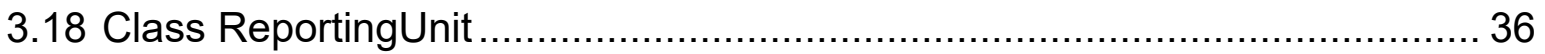

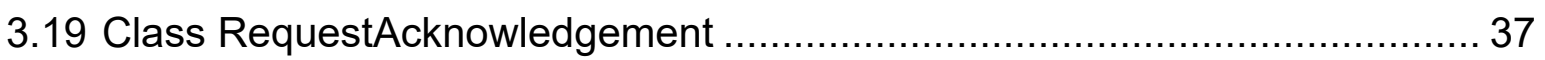

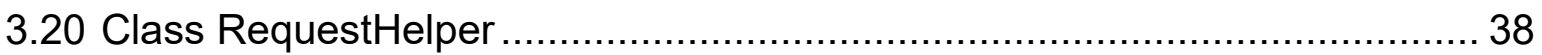

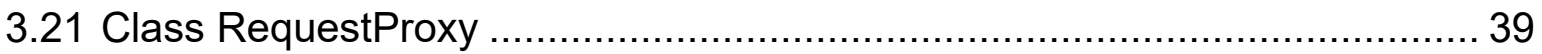

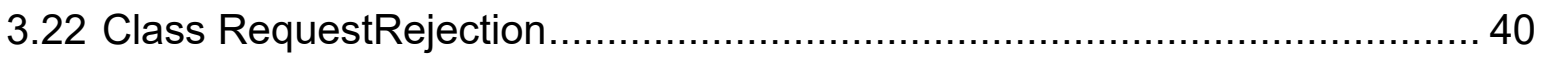

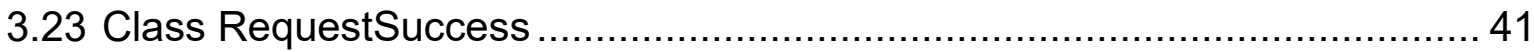

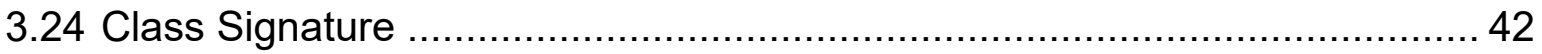

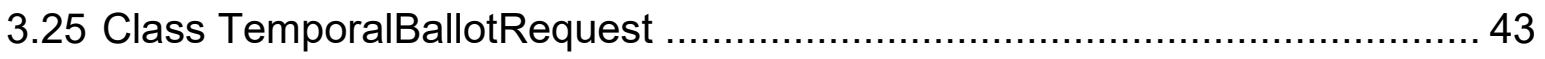

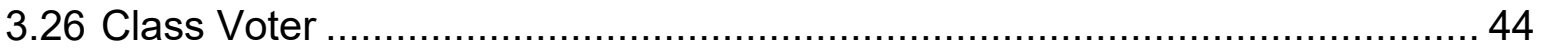

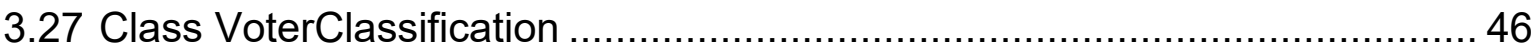

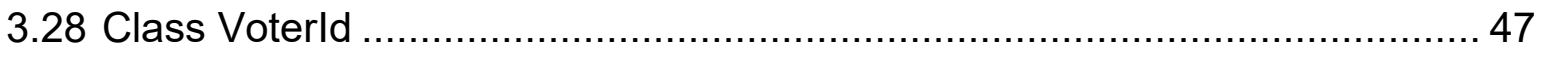

3.29 Class VoterParticipation......................................................................... 48

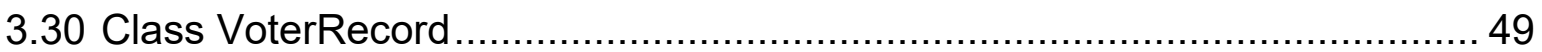

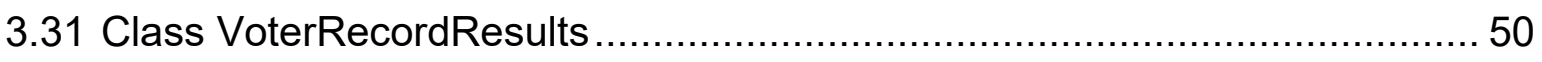

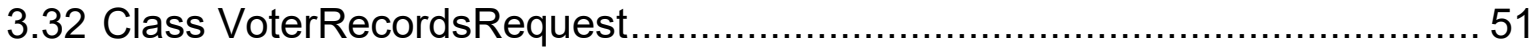

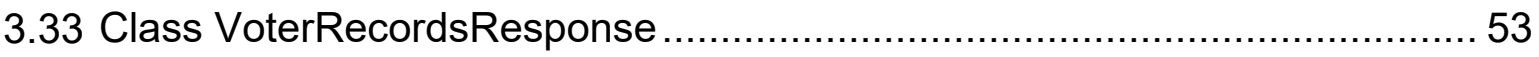

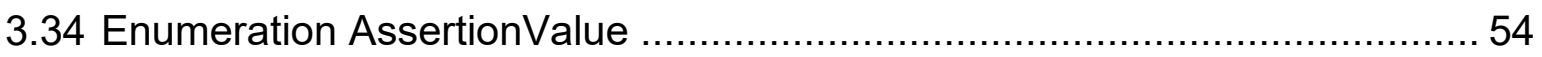

3.35 Enumeration BallotReceiptMethod ………...................................... 55

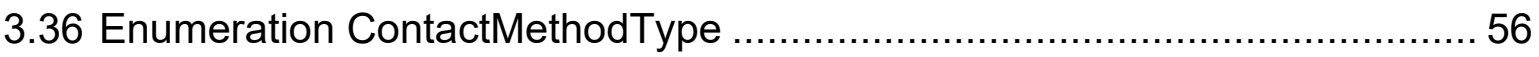

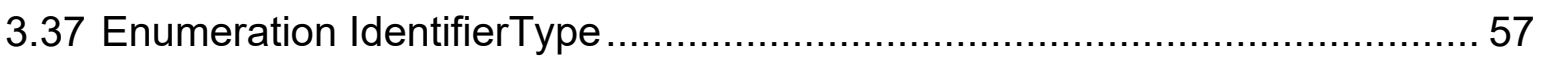

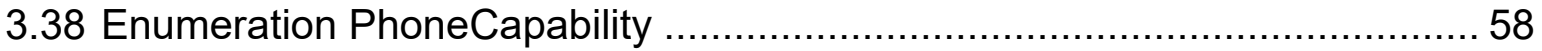

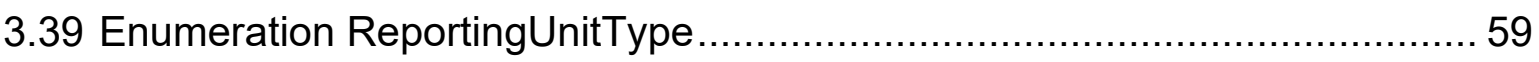

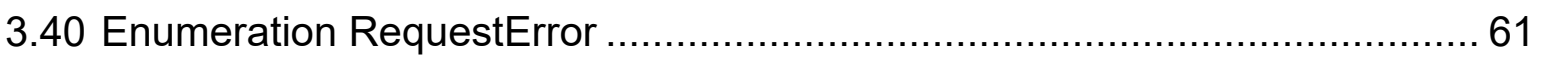

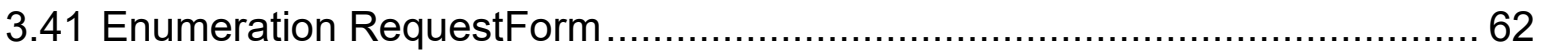

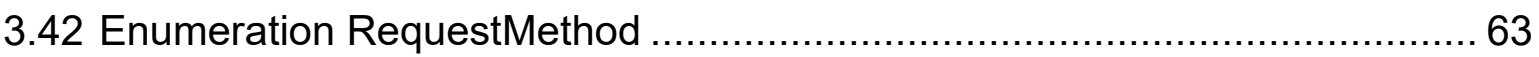

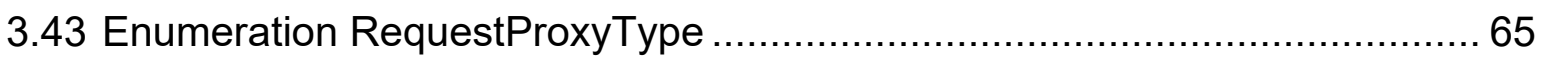

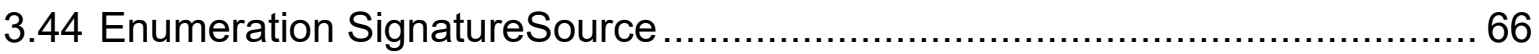




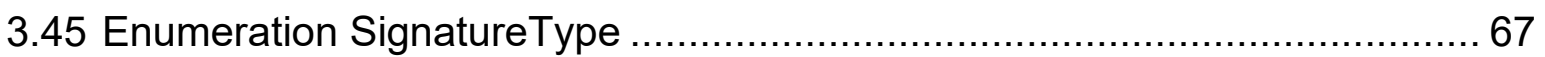

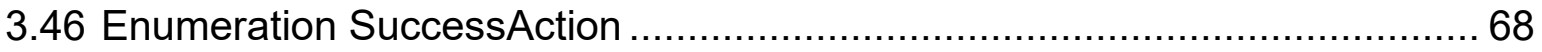

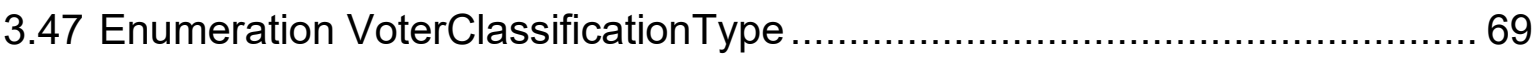

3.48 Enumeration VoterHelperType ........................................................ 71

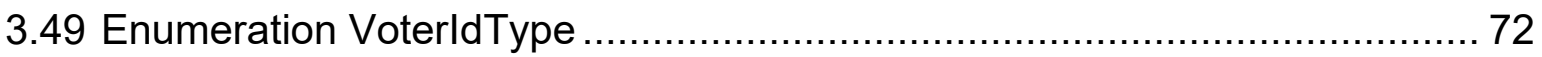

3.50 Enumeration VoterRequestType ......................................................... 73

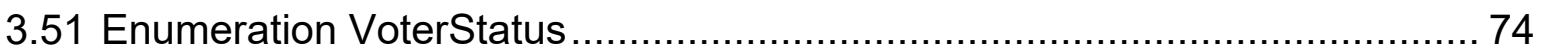

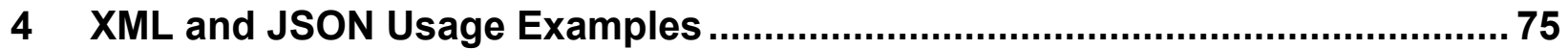

4.1 Example 1: NVRA-style Voter Registration Request in XML ..................... 75

4.2 Example 2: NVRA-style Voter Registration Request in JSON .................... 80

4.3 Example 3: NVRA-style Voter Records Response in XML …..................... 85

\section{List of Appendices}

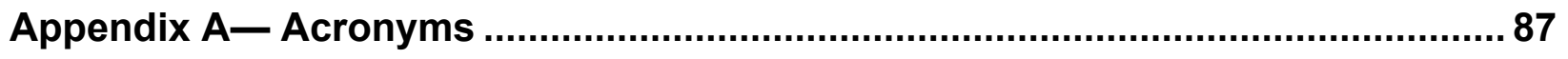

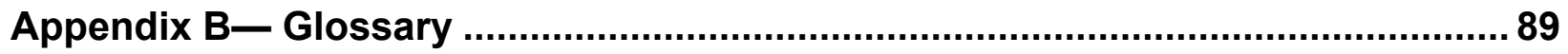

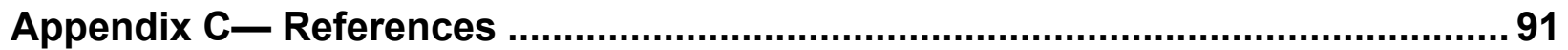

Appendix D— File Download Locations............................................................... 93

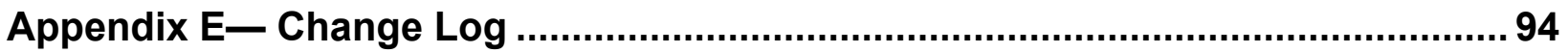




\section{Introduction}

This document is a specification for a common data format (CDF) for voter records data interchange related to voter registration, such as, registration or ballot requests from online voter portals made to voter registration (VR) systems, and responses to the requests returning from the VR system. The specification includes an XML (eXtensible Markup Language) [1] schema and references a JSON (JavaScript Object Notation) [2] schema.

The primary features of this specification include:

- A data format for voter registration requests and responses when using the National Voter Registration Act (NVRA) [3] or Federal Post Card Application (FPCA) [4] forms and state-specific variations of these forms.

- A data format for ballot requests and responses when using the FPCA forms, as well as state specific forms.

- A data model in Unified Modeling Language (UML) [5] that itemizes and defines the data involved in voter records interchange and that is used to derive the XML and JSON schemas.

- Instructions for implementing and using the XML and JSON schemas.

- A flexible specification to cover additional use cases for other types of registration transactions, such as for voter record maintenance.

\subsection{Why this Specification is Needed}

The purpose of this specification is to provide data interchange formats in XML and JSON for voter records requests and responses so as to assist election officials and developers in implementing and supporting the development of voter records systems within states. Advantages of using this specification include:

- Providing a ready data interchange format for OVR (on-line voter registration systems) to remove the need for individual system development projects to define their own data models and formats.

- Assisting election officials by reducing or eliminating non-standard exchange formats for voter registration data. Currently, the systems involved and data they produce do not interoperate, adding complexity to the process.

- Providing a baseline CDF (common data format) for voter registration data that can be continually refined to be more efficient and adaptable across all states. Once jurisdictions adopt the CDF voter records interchange (VRI) specification, their experience and feedback will refine the continued development of the specification.

- Providing the foundation for additional use cases in the future, which could include:

- matching driver's license data between the MVA and voter record systems,

0 automated notifications between the MVA and voter record systems,

o interoperability with electronic pollbooks, 
- voter record maintenance activities,

○ cross-state record matching, or

- facilitating data reporting for the Election Administration and Voting Survey (EAVS) [6].

\subsection{Intended Audience}

The intended audience of this specification includes election officials, VR system designers and developers, and others in the election community, including the general public. Some background in election administration and registration is useful in understanding the material in this specification.

\subsection{Motivation and Methodology}

This document was written primarily to assist election officials and developers in implementing and supporting voter record systems by reducing or eliminating non-standard exchange formats for voter record data. Additionally, there are sometimes significant variations among different jurisdictions within a state as well as among the states themselves in the way they automate voter registration and related parts of voter records management.

NIST and a community of U.S. election officials, analysts, and election system technologists analyzed varying VR scenarios and use cases and their associated data interchanges. They did this to analyze existing practices and create a standard data interchange format for emerging voter record systems. This specification implements the following use cases:

1. OVR Submission: Digital VR applications forms transmitted between components of state systems or to state systems by third-party systems, following the formats of the NVRA and FPCA voter registration application forms, including state-specific additions to these forms.

2. VR Update Submission: Similar application forms including voter registration updates (change of name, change of address), change of voter status, and ballot requests.

3. OVR Transfer: Subsets of applications used for third-party OVR assistant organizations to transfer users and user data to state OVR systems.

4. Voter Records Lookup: Requests for information regarding voter records within a VR system, or between a VR system and a third party.

A UML data model was subsequently generated to represent the data associated with voter record requests and responses and to show how the data elements are related and organized. Finally, XML and JSON schemas were generated from the UML data model.

The advantages of using a UML data model as an intermediate step to generating the XML and JSON schemas include that:

- the model is independent of the concrete data formats (or other potential formats that could be derived), and 
- relationships between data elements are easier to correctly define and visualize when they are independent of any specific data format.

If changes are needed to the specific XML and JSON formats, one can make changes to the UML model and then generate or derive new versions of the formats.

Much of the data involved in voter record requests consists of voter addresses such as current addresses, postal addresses, and previous addresses. Rather than implement new and complicated functionality in this specification for representing addresses, NIST decided to use the existing U.S. Thoroughfare, Landmark, and Postal Address Data Standard [7] which contains four major classes of addresses that are broken out into 11 different types of addresses. These can be used to represent addresses where voters live and where they receive postal mail (if different), including overseas addresses.

While this specification is focused on digital OVR submission, subsequent versions of this specification may implement additional use cases, including:

- MVA Match: Subsets of digital applications exchanged between state VR systems and MVA or similar systems, to perform driver's license data matching (if required) as part of OVR processing.

- MVA Notification: Data exchanged by MVA or similar systems and VR systems, as part of NVRA compliance to digitally notify VR systems of MVA records of its customers who requested voter registration. May also include similar data push from MVA of existing MVA records recently updated with change-of-address, as part of semiautomated steps toward permanent voter registration, or other forms of data exchange to VR systems that might facilitate elements of automatic or permanent voter registration.

- Cross-State Records Match: Data interchange between state VR systems and systems for records matching, for example the Electronic Registration Information Center (ERIC) [8].

- Election Administration Voting Survey (EAVS) Submission: Subsets of voter records externalized from voter records systems for data aggregation and reporting, including but not limited to EAVS reporting.

Note that this specification addresses U.S. governmental elections and is not intended for use "as is" in other types of elections or in other countries. However, the specification was written with the intention that it be adaptable to other election environments.

\subsection{Document Structure}

This specification includes the following material:

- Section 2 - overview of the supported voter record transactions, the VRI UML model, and the FGDC (Federal Geographic Data Committee) standard.

- Section 3 - VRI UML Model documentation. 
- Section 4 - VRI XML and JSON usage examples, including registration requests and responses.

- Appendices - Acronyms, glossary, references, and schema download locations. 


\section{Overview of Digital OVR Transactions}

This section presents an overview of the digital OVR voter registration transactions supported by this specification and examples of how they are implemented using the NVRA and FPCA forms (shown in Figures 1 and 2) as well as with state-specific forms, e.g., modifications to the NVRA. It also contains an overview of the U.S. Thoroughfare, Landmark, and Postal Address data standard [7] used by this specification for representing voter addresses.

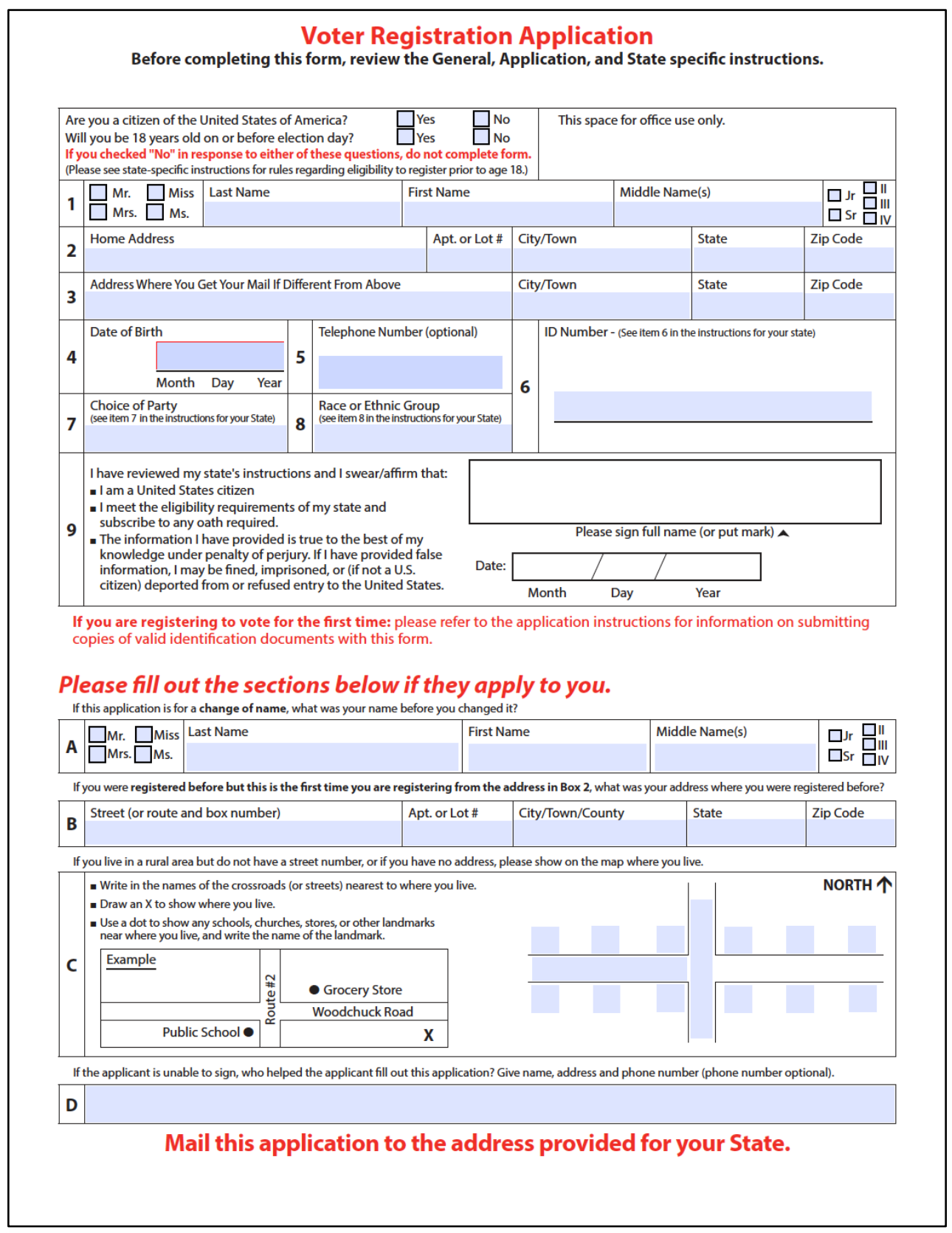

Figure 1 - National Voter Registration Act (NVRA) form 


\section{Voter Registration and Absentee Ballot Request}

Federal Post Card Application (FPCA)

Print clearly in blue or black ink. 1. Who are you? Pick one.

口 I am on active duty in the Uniformed Services or Merchant Marine -OR- $\square$ I am an eligible spouse or dependent. I request an absentee
$\square$ am on active duty in the Uniformed Services or Merchant

in which I am eligible

to vote AND:

I am a U.S. citizen living outside the country, and I intend to return.

I am a U.S. citizen living outside the country, and my return is uncertain.

I am a U.S. citizen living outside the country, and I have never lived in the United States.

Last name

First name

Middle name

Social Security Number

Suffix (Jr., II)
Previous names (if applicable)

2. What is your address in the U.S. State or ternitory where you are registering to vote and requesting an absentee ballot?

Your voting materials will not be sent to this address. See instructions on other side of form.

Street address

City, town, village

County

ZIP

\section{Where are you now? You must give your CURRENT address to receive your voting materials.}

Your mailing address. (Different from above) Your mail forwarding address. (If applicable)

\section{What is your contact information? This is so election officials can reach you about your request.}

Provide the country code and area code with your phone and fax number. Do not use a Defense Switched Network (DSN) number.

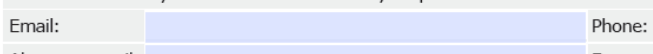

Alternate email: $\quad$ Fax:

\section{What is your voting preference? Select One.}

How do you want to $\square$ Mail

to

from your election office? $\square$ Fax

\section{What additional information must you provide?}

The following need more information: Alaska, Arizona, Puerto Rico, Vermont, and Virginia. (Ex. Proof of residency, employer, etc.) You may also use this space to clarify your voter information. See the Voting Assistance Guide at FVAP.gov.

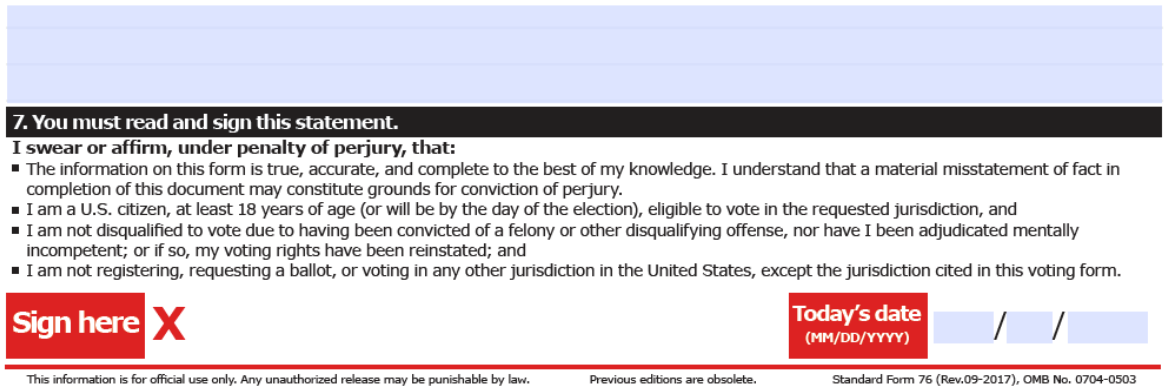

Figure 2 - Federal Post Card Application (FPCA) form

The forms shown in Figures 1 and 2 are paper-based, whereas states implementing OVR make digital representations of the forms, usually with some state-specific modifications that may request additional data. 


\subsection{Voter Records Request}

The digital NVRA and FPCA forms form the basis for digital OVR registration. The NVRA form is used for U.S. non-military citizen registrations whereas the FPCA form is used by U.S. military, their families, and citizens residing outside the U.S. to register and, if desired, request a ballot at the same time.

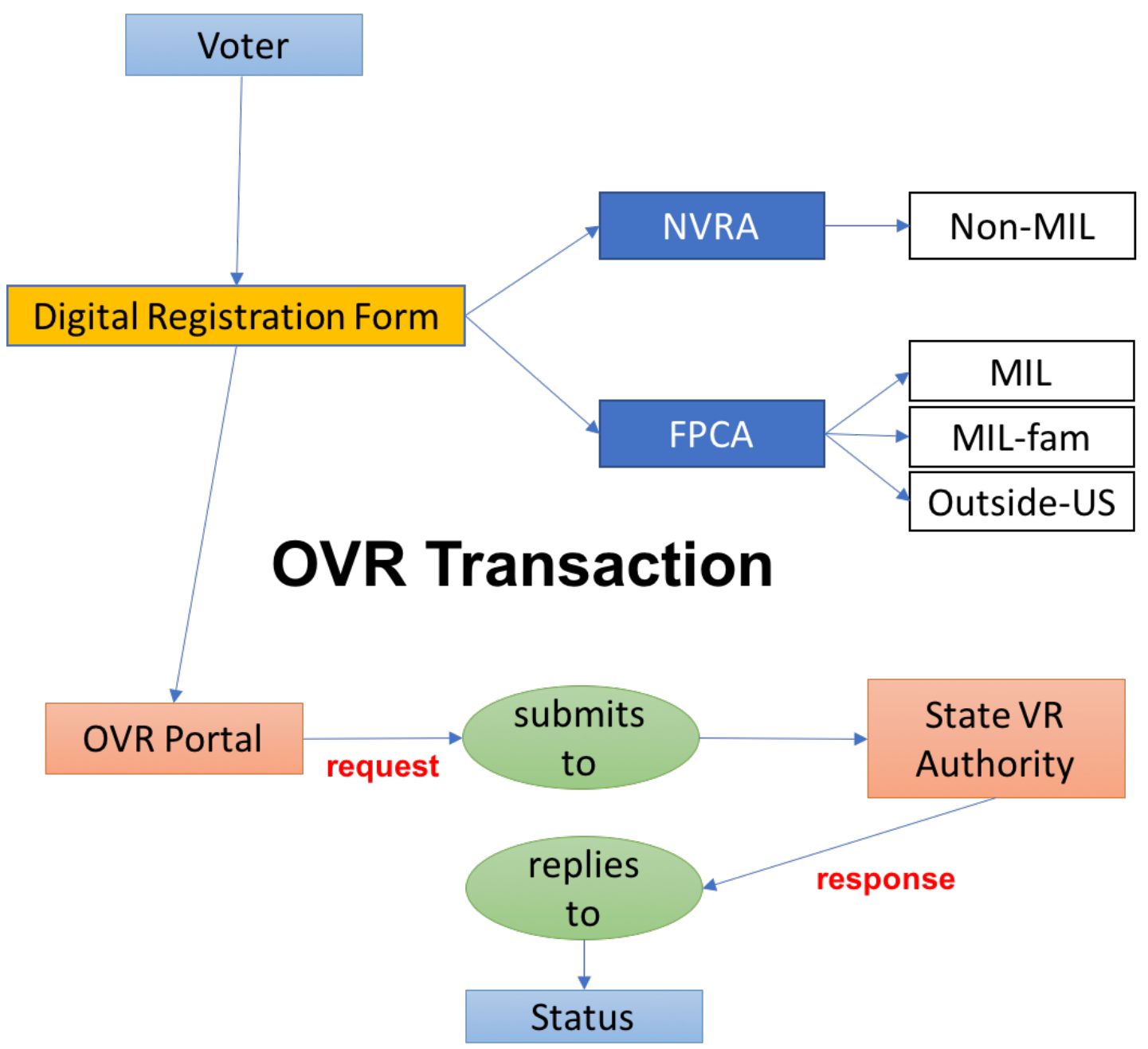

Figure 3 - Voter Registration Use Case

The submission of a digital registration form to a VR authority represents a voter records request. The response transmitted back from the VR authority to the submitter includes a status such as "registration-created" or would indicate an error for reasons including "incomplete" or "ineligible". The request consists of a registration request, e.g., initial registration, followed by various information about the voter and the submitter of the request. 
The use case for the digital OVR request, shown in Figure 3, includes:

- A client IT "OVR submitter",

- A service IT system "VR authority", and

- The submission, a digital NVRA, FPCA, or otherwise state-specific form via transmission from OVR submitter to VR authority.

Currently, VR authorities are typically the back-end components of actual state OVR systems, while submitters include clients of state OVR systems such as the MVA or other clients that are operated by third-party VR organizations and that integrate with OVR systems by sending all or part of an NVRA/FPCA dataset collected by the client from a human registrant.

An OVR transaction, as used in this specification, generally consists of a voter records request followed by a voter records response, although this may vary across U.S. states and territories. The voter records request UML model in Figures 4 and 5 include four types of requests that could be sent from an OVR submitter to a VR system (see the VoterRequestType enumeration in Figure 5):

1. Request a registration for a voter using the digital NVRA or FPCA form.

2. Request a lookup for one or more voter registration records.

3. Request a ballot for one more elections as part of the digital FPCA form, or as part of a state specific digital form.

4. Request a state-specific action for a voter using possibly a state-specific digital form.

The Voter class is the primary class; it contains information about the voter, including name, addresses, party registration, voter's signature, method of contact, etc. If a third-party registration assistant or proxy is being used, the RequestHelper or RequestProxy classes are used to include name and other information generally required by the registration forms.

Both the voter records request and response models contain a class ExternalIdentifier, which is used to associate an identifier with an item. In the case of the request model, it is used optionally to associate an identifier to the political party, and in the response model, to optionally associate identifiers with political geography such as precincts and districts. The enumerations often contain a value called "other", which is to be used when none of the enumeration values apply. Classes that use these enumerations generally contain an OtherType or similarly-named attribute to contain that value. For example, in the ContactMethod class, if none of the enumeration values of ContactMethodType apply, "other" would be used in the Type attribute, and the OtherType attribute would contain the value 


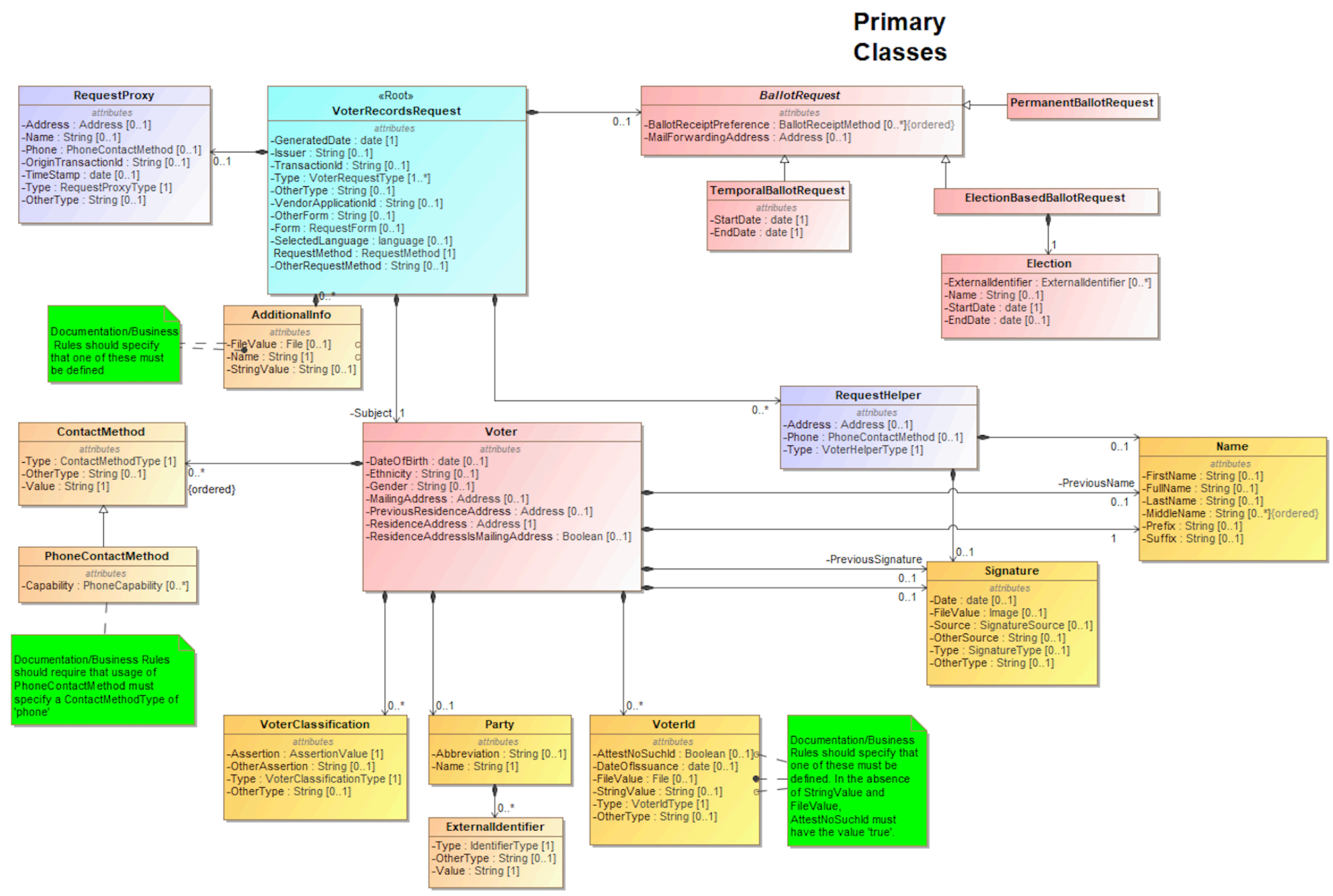

Figure 4 - Voter Records Request UML Class Diagram 


\section{Enumerations}

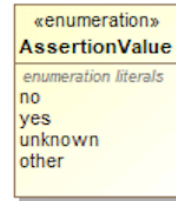

\begin{tabular}{|l|}
\multicolumn{1}{c|}{ «enumeration» } \\
BallotReceiptMethod \\
\hline \multicolumn{1}{|c|}{ enumeration fiterals } \\
email \\
email-or-online \\
fax \\
mail \\
online \\
\hline
\end{tabular}

\begin{tabular}{l} 
«enumeration» \\
ContactMethodType \\
enumeration Ifterals \\
email \\
phone \\
other \\
\hline
\end{tabular}

\begin{tabular}{|l|}
\hline «enumeration» \\
IdentifierType \\
\hline fips \\
local-level \\
national-level \\
ocd-id \\
state-level \\
other \\
\hline
\end{tabular}

\begin{tabular}{|l|}
\hline \multicolumn{1}{|c|}{ «enumeration» } \\
PhoneCapability \\
\hline enumeration literals \\
fax \\
mms \\
sms \\
voice \\
\hline
\end{tabular}

\begin{tabular}{|l|}
\hline \multicolumn{1}{|c|}{ «enumeration» } \\
RequestForm \\
\hline enumeration literals \\
fpca \\
nvra \\
other \\
\hline \hline
\end{tabular}

\begin{tabular}{|l|}
\hline \multicolumn{1}{|c|}{ «enumeration» } \\
VoterHelperType \\
\hline $\begin{array}{l}\text { enumeration literals } \\
\text { assistant } \\
\text { witness }\end{array}$ \\
\hline
\end{tabular}

\begin{tabular}{|c|}
\hline $\begin{array}{l}\text { «enumeration» } \\
\text { RequestMethod }\end{array}$ \\
\hline $\begin{array}{l}\text { enumeration literals } \\
\text { armed-forces-recruitment-office } \\
\text { motor-vehicle-office } \\
\text { other-agency-designated-by-state } \\
\text { public-assistance-office } \\
\text { registration-drive-from-advocacy-group-or-political-party } \\
\text { state-funded-agency-serving-persons-with-disabilities } \\
\text { voter-via-election-registrars-office } \\
\text { voter-via-email } \\
\text { voter-via-fax } \\
\text { voter-via-internet } \\
\text { voter-via-mail } \\
\text { unknown } \\
\text { other }\end{array}$ \\
\hline
\end{tabular}

\begin{tabular}{|c|}
\hline $\begin{array}{c}\text { «enumeration» } \\
\text { RequestProxyType }\end{array}$ \\
\hline $\begin{array}{l}\text { enumeration literals } \\
\text { armed-forces-recruitment-office } \\
\text { motor-vehicle-office } \\
\text { other-agency-designated-by-state } \\
\text { public-assistance-office } \\
\text { registration-drive-from-advocacy-group-or-political-party } \\
\text { state-funded-agency-serving-persons-with-disabilities } \\
\text { other }\end{array}$ \\
\hline
\end{tabular}

«enumeration» VoterClassificationType enumeration fiterals
activated-national-guard active-duty

active-duty-spouse-or-dependent citizen-abroad-intent-to-return citizen-abroad-return-uncertain citizen-abroad-never-resided deceased

declared-incompetent eighteen-on-election-day \begin{tabular}{|l|}
\hline «enumeration» \\
Signature Type \\
\hline enumeration litera/s \\
dynamic \\
electronic \\
other
\end{tabular}

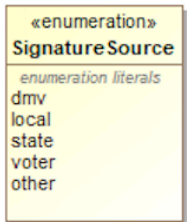

permanently-denied

protected-voter

united-states-citizen other

\begin{tabular}{|l|}
\hline \multicolumn{1}{|c|}{\begin{tabular}{c|}
\multicolumn{1}{|c|}{ "enumeration» } \\
VoterldTyperation fiterals
\end{tabular}} \\
\hline drivers-license \\
local-voter-registration-id \\
ssn \\
ssn4 \\
state-id \\
state-voter-registration-id \\
unspecified-document \\
unspecified-document-with-name-and-address \\
unspecified-document-with-photo-identification \\
unknown \\
other
\end{tabular}

Figure 5 - Voter Records Request UML Enumerations 


\subsection{Voter Records Response}

The voter records response simply returns a response to the voter records request. The UML model is simpler than the request model in that a response generally contains little data other than the results of the request, which are:

1. The registration request was acknowledged, but no further status is available.

2. The registration request was rejected, including a reason(s) for the rejection.

3. The registration request succeeded, including the action(s) taken.

The response model also allows for a set of voter records to be returned, if the request Type was set to lookup.

The UML model in Figure 6 shows the VoterRecordsResponse class, which has four corresponding types of responses. The first, RequestAcknowledgement, represents the typical current practice in online voter registration, where a registration authority operates an online service that receives VR requests and saves them for later processing by local elections offices' staff. In this typical practice, the acceptance of an online VR request is simply an acknowledgement that the request was received without error.

The second type of response, RequestRejection, contains the reason(s) for the error that is causing the rejection as well as any additional details. While the various errors that can occur are beyond the scope of this specification, the RequestError enumeration lists a series of common errors, including:

1. The request is incomplete in some way including incomplete address or name or birthdate or signatures.

2. A lookup of the voter's identity failed.

3. The voter is ineligible to vote.

If none of the errors in the enumeration are appropriate, a different error can be specified in the OtherError attribute.

The third type of response, RequestSuccess, is used for cases where the receiving service is able to process a request to success and notify the sender synchronously. The contents of a RequestSuccess are modeled on the information that becomes available to a newly registered voter as a result of successful registration.

In the UML model, the RequestSuccess class optionally includes these items: voter's assigned polling place and precinct, the location of the local election authority, and a list of districts that the voter resides in. The successful registration also returns the registration action(s) that occurred, which may differ from what was requested. For example, a request for a new voter registration may succeed, but if the voter was already registered, the response may indicate a registration update as opposed to a registration creation. 


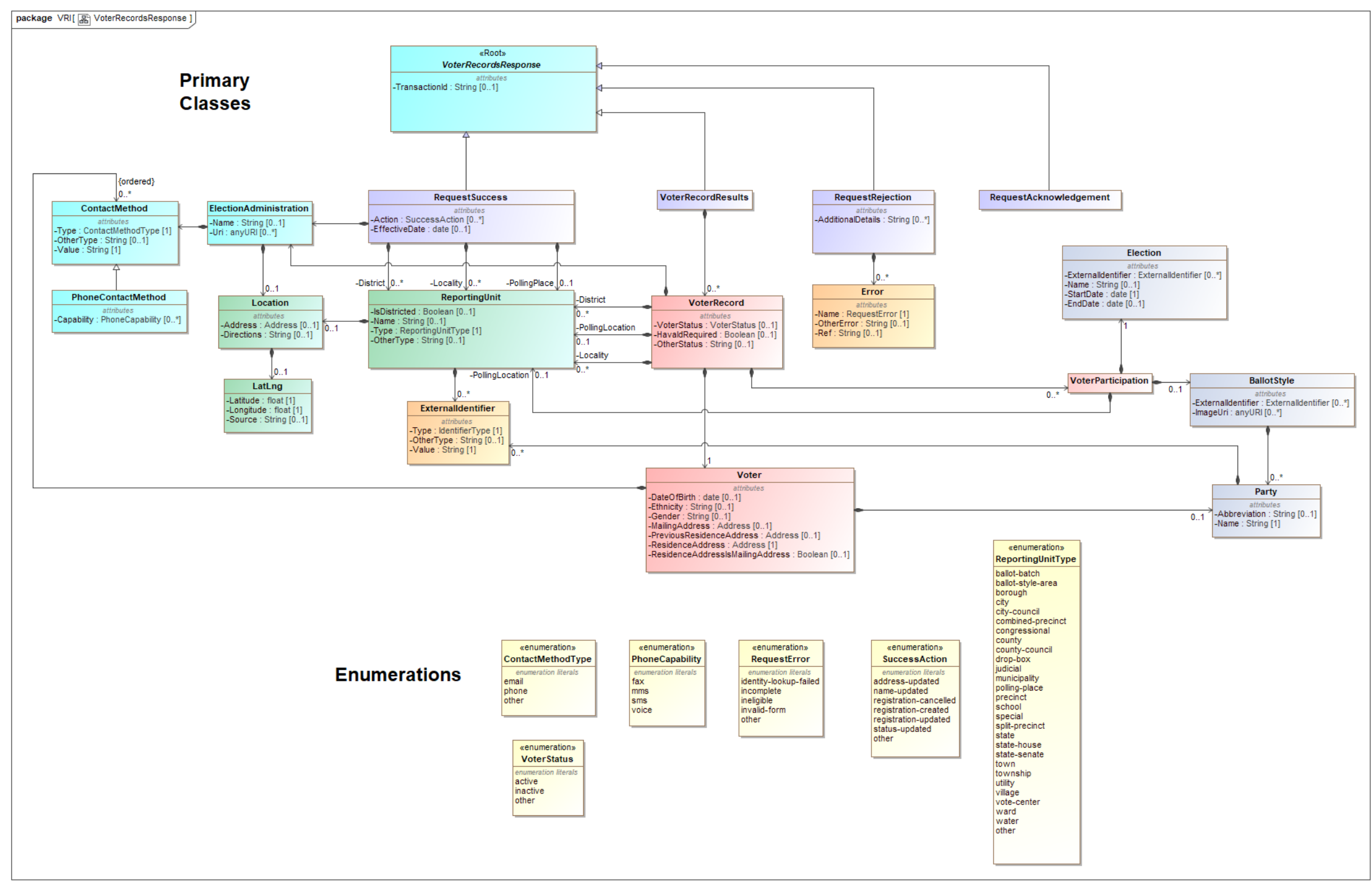

Figure 6 - Voter Records Response UML Class Diagram 
The fourth type of response, VoterRecordResults, is used when responding to the specific request of a lookup of one or more voter's records. The contents of VoterRecordResults includes a VoterRecord for each potential match.

Each VoterRecord contains details about the voter's registration and optionally information regarding the voter's election participation history, voter's assigned polling place and precinct, the location of the local election authority, and a list of districts that the voter resides in.

\subsection{Including State-specific Request and Response Items}

The UML model and associated XML and JSON schemas contain several features to enable state-specific requests and responses. Using these features, it is possible to use this specification as local modifications are needed.

The AdditionalInfo class is used for information not addressed in this schema by other attributes, e.g., state-specific data that does not "fit" in any other attribute. The type of data will thus be highly specific to the generating application, and consuming applications must "know" the meaning of the data to make use of it. Each AdditionalInfo class would contain the name of the data, and then its value, depending on whether the data represents the value directly as a string or represents a file name.

For example, if a state requires its registration form to include the voter's language, it would need to use the AdditionalInfo class to contain a value representing the voter's language. An $\mathrm{XML}$ example is as follows, using English as the voter's language:

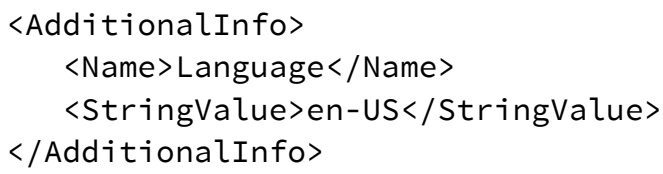

The XML and JSON usage examples in Section 4 contain a number of examples showing usage of the AdditionalInfo class. Additionally, each enumeration generally contains an "other" value that can be used when none of the enumeration values are sufficient. If "other" is used as the enumeration value, there is an attribute named OtherType that can be used to hold the other data. For example, a state may wish to implement a specific "address-update" transaction and would thus need to use "other" for the Type attribute in the VoterRecordsRequest class. The OtherType attribute would then contain the type of registration request, i.e., "address-update", e.g. in XML,

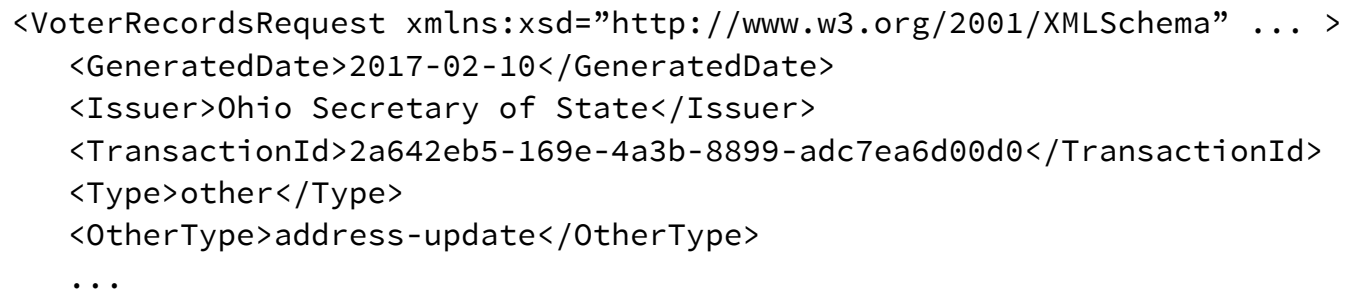




\subsection{Dealing with Addresses - The U.S. Thoroughfare, Landmark, and Postal Address Data Standard}

Voter addresses are perhaps the most complex part of a voter registration request or other related data exchanges. As you will see in the examples in this specification, the vast majority of the data in a voter registration request has to do with voter addresses. There are multiple types of voter addresses for VR purposes, e.g.:

- Current registration address,

- Previous registration address,

- Multiple types of addresses for location and mailing purposes, e.g.,

- Postal mailing address,

- Structured and unstructured street address,

○ Rural addresses,

- PO (postal office) box addresses,

- Military and diplomatic addresses, and

○ Mailing addresses outside the U.S.

Rather than "re-inventing the wheel" by specifying an address format, this specification makes use of an existing XML-based standard for structuring addresses: the U.S. Thoroughfare, Landmark, and Postal Address Data Standard [7] issued by the Federal Geographic Data Committee (FGDC) [9] and covering the complexity of addresses managed by or encountered by organizations and agencies such as the United States Census and USPS (U.S. Postal Service). Use of the FGDC standard greatly simplifies this specification and leaves maintenance of the standard to a more appropriate management body.

A complete overview of the FGDC standard and how to use it is beyond the scope of this specification, and readers and developers are encouraged to refer to the FGDC standard documentation. Briefly, the FGDC standard classifies all U.S. addresses into a simple taxonomy of address classes organized into 4 groups:

1. Thoroughfare Classes -5 address types,

2. Landmark Classes - 2 address types,

3. Postal Deliver Classes - 3 address types, and

4. General Class - 1 address type.

Altogether, there are a total of 11 address types (most are Thoroughfare types, see section 2.4.1 below). The address types are used, then, for voter addresses in the VRI specification, which includes the following four types of voter addresses:

- ResidenceAddress

- PreviousResidenceAddress,

- MailingAddress, and

- MailForwardingAddress. 
To deal with these voter addresses in a consistent manner, the XML and $\mathrm{JSON}^{1}$ schemas generated from the UML model map the <Address $>$ type to one of the 11 different address types in the FGDC schema, as shown in Figure 7. Note that a namespace prefix of addr is used for XML types corresponding to the 11 FGDC address types; addr_type is also used for other address component types that are defined in a schema included by the FGDC schema (shown in other examples in this specification).

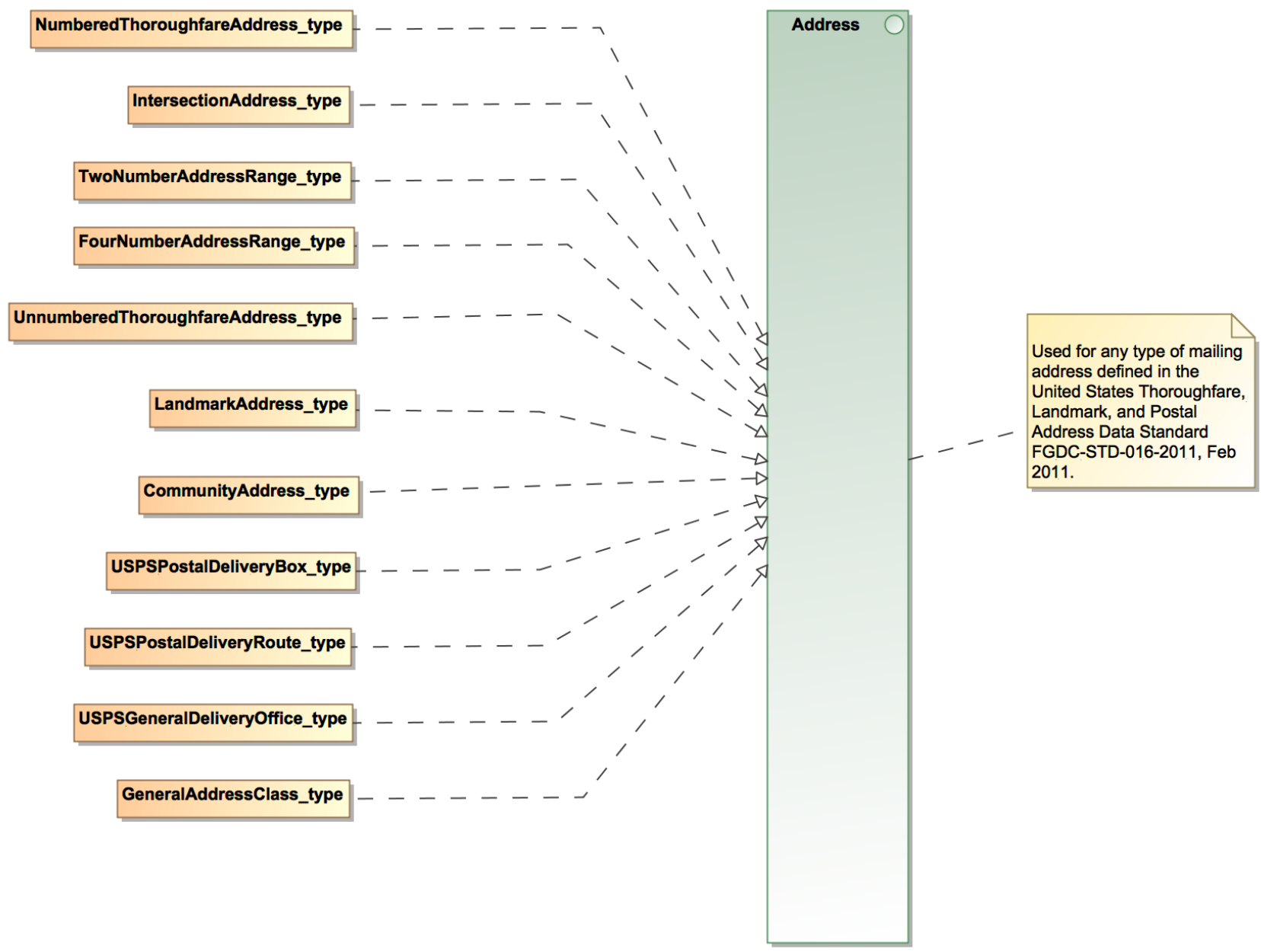

Figure 7 - FGDC Address Types Mapped to Single Address Attribute

\subsubsection{Thoroughfare Classes}

Most business and residential addresses are Thoroughfare addresses; therefore this class will be used most often. It specifies a location by reference to a thoroughfare, i.e., a road or part of a road or other access route along which a delivery point can be accessed. A thoroughfare is

\footnotetext{
${ }^{1}$ Because the FGDC standard was written for XML and does not include a JSON schema, NIST created a JSON version from the relevant sections of the FGDC XML schema (see Appendix D for download locations).
} 
typically but not always a road - it may be, for example, a walkway, a railroad, or a river. The thoroughfare address types are:

1. Numbered Thoroughfare Address, e.g., 123 Main Street.

2. Intersection Address, e.g., Fifth Avenue and Main Street.

3. Two Number Address Range, e.g., 405-411 West Green Street.

4. Four Number Address Range, e.g., 900-962, 901-963 Milton Street.

5. Unnumbered Thoroughfare Address, e.g., Forest Service Road 698.

Of the 5 types listed, Thoroughfare Addresses are generally used most often. Unnumbered Thoroughfare Addresses are also used for those areas where no address numbers have been assigned and the addresses often include only the thoroughfare name. A simple XML example showing the Numbered Thoroughfare type used in the <MailingAddress> element for "500 W Tuscarawas Ave, Barberton, $\mathrm{OH} 44203$ " is as follows:

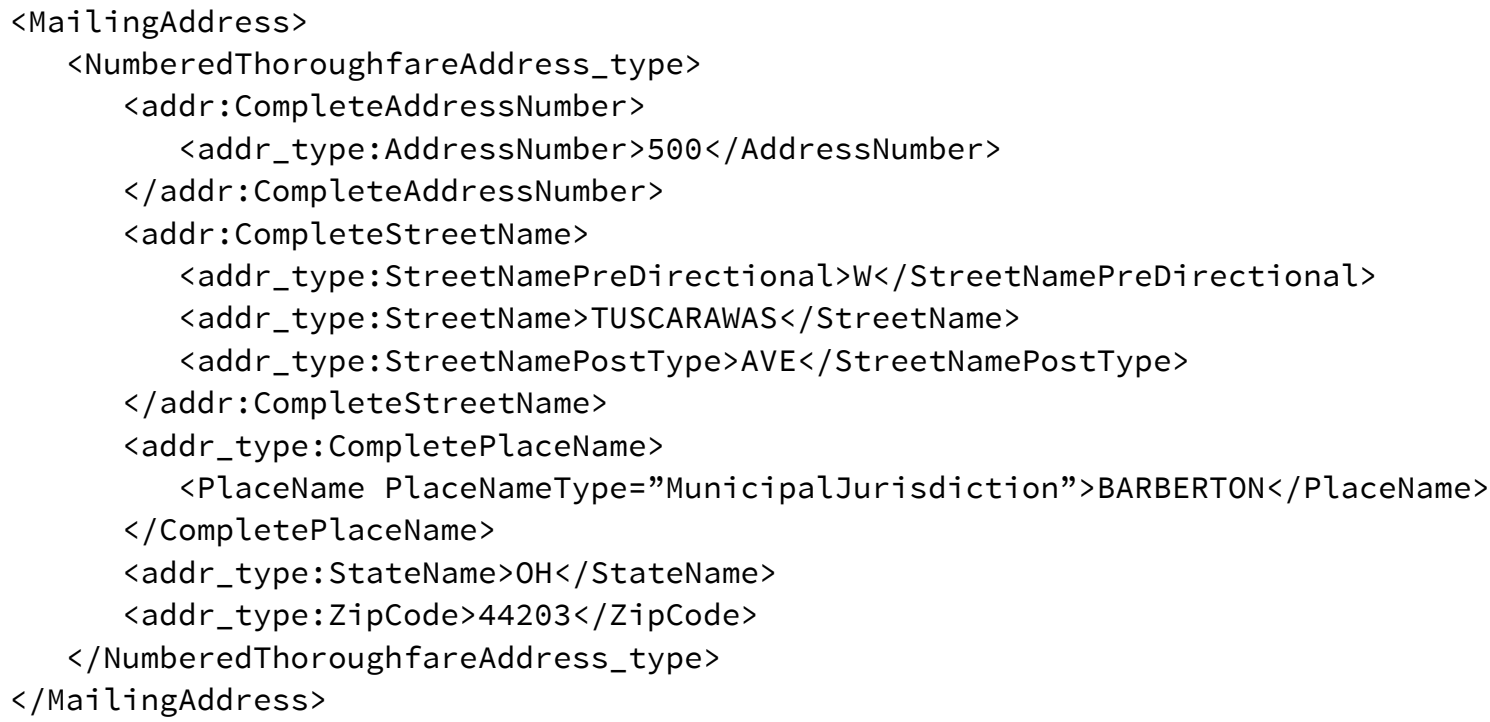

\subsubsection{Landmark Classes}

Landmark addresses specify a location by reference to a named landmark. A landmark is a relatively permanent feature of the manmade landscape that has recognizable identity within a particular cultural context, e.g., a large statue or structure such as an apartment complex. The landmark address types are:

- Landmark Address, e.g., Statue of Liberty.

- Community Address, e.g., 123 Urbanizacion Los Olmos, Ponce, PR 00731-1235. 


\subsubsection{Postal Delivery Classes}

Postal delivery addresses specify points of postal delivery that have no definite relation to the location of the recipient, such as a post office box, rural route box, overseas military address, or general delivery office. The USPS specifies each class in detail in USPS Publication 28[10]. The postal delivery types are:

- USPS Postal Delivery Box, e.g., PO Box 16953.

- USPS Postal Delivery Route, e.g., RR 1, Box 100.

- USPS General Delivery Office, e.g., General Delivery.

\subsubsection{General Address Class and Handling Non-U.S. Addresses}

The general address class provides a "catch-all" way to handle addresses, including non-U.S. addresses. The general address class may include addresses from any or all of the other classes, or addresses whose class is unknown or whose syntax does not conform to any of the thoroughfare, landmark, and postal classes. Although the scope of the FGCD standard is restricted to U.S. addresses, this class was included to facilitate reconciliation with address standards of other nations and to accommodate files that mix addresses from the U.S. and other countries.

There are three types mapped to this class:

- The complete address as a single unparsed string of text, e.g., PO Box 1511, Ames, IA 50010.

- $\quad$ The complete address with place, state and zip code parsed out to a single field, e.g., $P O$ Box 1511; Place State ZIP = Ames, IA 50010.

- The complete address with place, state, zip code, zip plus 4, and country name are parsed out to separate fields, e.g., PO Box 1511; Complete Place Name = Ames; State Name = IA; Zip Code = 50010; Country Name $=$ USA

ISO 3166-1 [12] country codes is favored for country names, e.g., USA for United States, MEX for Mexico, GBR for United Kingdom.

A simple XML example for "PO Box 1511, Ames, IA 50010-4231, USA" in which the address components are structured to the extent possible is as follows:

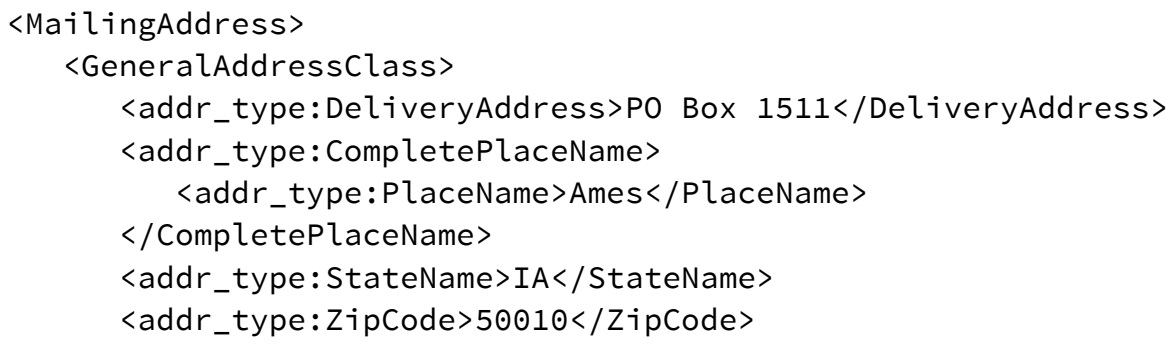




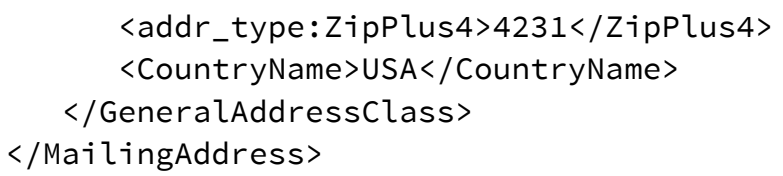

For non-U.S. or overseas addresses, it may or may not be practical or possible to structure the address similarly. A simple XML example is as follows for "33, boulevard du Port, F 95510 Cergy-Pontoise cedex, France":

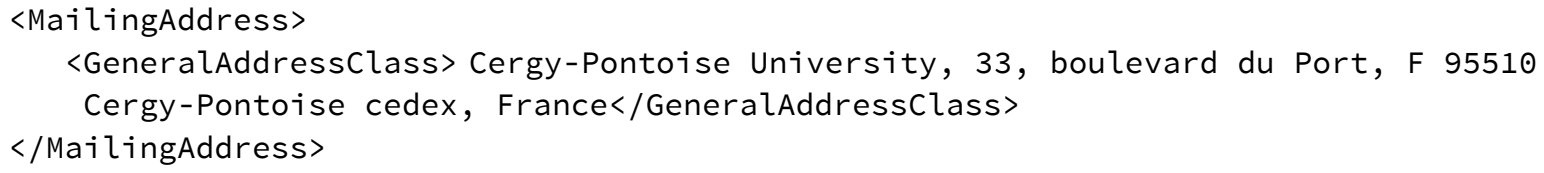

The entire address is contained in an unstructured string, however it is possible to specify it with more structure, as follows:

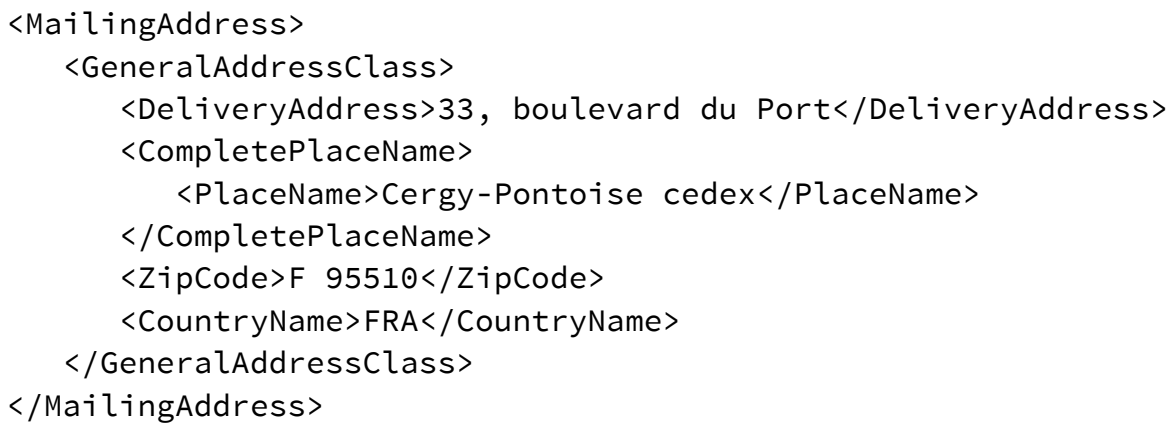

For additional usage information, the FGDC standard documentation should be consulted [6]. 


\section{Voter Records Interchange UML Model Documentation}

This section contains documentation for each of the UML classes, attributes, and enumerations. Curly braces around an attribute indicate that the attribute is a reference to an instance of an associated class. For example, the attribute $\{$ GpUnit $\}$ is a reference to an instance of the GpUnit class.

\subsection{Class Additionallnfo}

Used in request messages for specifying information not addressed in this model by other attributes, e.g. state-specific information that does not "fit" in any other attribute. The information will thus be highly specific to the generating application, and consuming applications must "know" the meaning of the information to make use of it. For this reason, use of this class is discouraged as much as is possible.

The StringValue and FileValue attributes are both optional, however exactly one of them must be included.

\begin{tabular}{|l|}
\hline \multicolumn{1}{|c|}{ Additionallnfo } \\
\hline \multicolumn{1}{|c|}{ attributes } \\
-FileValue : File [0..1] \\
-Name: String [1] \\
-StringValue : String [0..1] \\
\hline \hline
\end{tabular}

Figure 8 - Additionallnfo

\begin{tabular}{|l|c|c|l|}
\hline \multicolumn{1}{|c|}{ Attribute } & Multiplicity & Type & \multicolumn{1}{c|}{ Attribute Description } \\
\hline FileValue & $0 . .1$ & File & $\begin{array}{l}\text { Used if the value is in a file; contains the filename and } \\
\text { MIME (Multipurpose Internet Mail Extension) type }\end{array}$ \\
\hline Name & 1 & String & Name of the value. \\
\hline StringValue & $0 . .1$ & String & Used if the value is a string; contains the string. \\
\hline
\end{tabular}




\subsection{Class BallotRequest}

An abstract class, used in request messages for a request for a ballot. Classes for specific types of BallotRequest inherit the attributes and define their own.

\begin{tabular}{|c|c|}
\hline BallotRequest \\
\hline atthibutes \\
-BallotReceiptPreference : BallotReceiptMethod [0..* ${ }^{*}\{$ ordered\} \\
-MailForwardingAddress : Address [0..1] \\
\hline \hline
\end{tabular}

Figure 9 - BallotRequest

\begin{tabular}{|l|l|l|l|}
\hline Attribute & Multiplicity & Type & Attribute Description \\
\hline BallotReceiptPreference & $0 . .^{*}$ & BallotReceiptMethod & $\begin{array}{l}\text { The voter's preference on } \\
\text { how to receive their ballot in } \\
\text { order from their most } \\
\text { preferred method to least. If } \\
\text { omitted, the default method } \\
\text { for the form will be used. }\end{array}$ \\
\hline MailforwardingAddress & $0 . .1$ & Address & \\
\hline
\end{tabular}




\subsection{Class BallotStyle}

Used in response messages for referencing a ballot style defined elsewhere, such as in an Election Management System (EMS).

\begin{tabular}{|c|}
\hline BallotStyle \\
\hline attributes \\
-Externalldentifier : Externalldentifier [0.. $\left.{ }^{*}\right]$ \\
-ImageUri : anyURI [0.."]
\end{tabular}

Figure $10-$ BallotStyle

\begin{tabular}{|l|c|c|l|}
\hline \multicolumn{1}{|c|}{ Attribute } & Multiplicity & \multicolumn{1}{|c|}{ Type } & \multicolumn{1}{|c|}{ Attribute Description } \\
\hline ExternalIdentifier & $0 . *^{*}$ & $\begin{array}{l}\text { ExternalIdentifier } \\
\text { for associating an ID with the } \\
\text { ballot style. }\end{array}$ \\
\hline ImageUri & $0 . .^{*}$ & anyURI & $\begin{array}{l}\text { URI (Uniform Resource Identifier) } \\
\text { for a ballot image. }\end{array}$ \\
\hline$\{$ Party\} & $0 . *^{*}$ & Party & $\begin{array}{l}\text { For associating one or more } \\
\text { parties with the ballot style. }\end{array}$ \\
\hline
\end{tabular}




\subsection{Class ContactMethod}

Used in request and response messages in three ways:

1. ElectionAdministration optionally includes this class to specify how to contact the election administration.

2. Voter optionally includes this class to specify the method for contacting a voter regarding the voter's request. If the voter can be contacted in multiple ways, the application creating the data should order the occurrences of ContactMethod by priority.

3. The PhoneContactMethod class uses ContactMethod as a base class, and should be used with when the contact method is for a telephone and it is necessary to describe the capabilities of the telephone.

The Capability attribute is provided by the PhoneContactMethod class.

\begin{tabular}{|l|}
\hline \multicolumn{1}{|c|}{ ContactMethod } \\
\hline attnbutes \\
-Type : ContactMethodType [1] \\
-OtherType : String [0..1] \\
-Value : String [1]
\end{tabular}

Figure 11 - ContactMethod

\begin{tabular}{|l|c|c|l|}
\hline \multicolumn{1}{|c|}{ Attribute } & Multiplicity & \multicolumn{1}{|c|}{ Type } & \multicolumn{1}{|c|}{ Attribute Description } \\
\hline Type & 1 & ContactMethodType & $\begin{array}{l}\text { The contact method type, e.g. email or } \\
\text { phone. }\end{array}$ \\
\hline OtherType & 0.1 & String & $\begin{array}{l}\text { Used if ContactMethodType value is } \\
\text { other. }\end{array}$ \\
\hline Value & String & $\begin{array}{l}\text { The value of the ContactMethod. This } \\
\text { will be the text value of the phone } \\
\text { number, email address, or other } \\
\text { mechanism. The values must be free of } \\
\text { any formatting characters, such as } \\
\text { parentheses or dashes for a phone } \\
\text { number. }\end{array}$ \\
\hline
\end{tabular}




\subsection{Class Election}

Used in request and response messages to describe an election event. Only the date of the election is required. Other attributes may be used to describe the election for which a ballot is requested or a voter participated.

\begin{tabular}{|l|}
\hline \multicolumn{1}{|c|}{ Election } \\
\hline attributes \\
-Externalldentifier : Externalldentifier [0..* $]$ \\
-Name : String [0..1] \\
-StartDate : date [1] \\
-EndDate : date [0..1] \\
\hline
\end{tabular}

Figure 12 - Election

\begin{tabular}{|l|c|c|l|}
\hline \multicolumn{1}{|c|}{ Attribute } & Multiplicity & \multicolumn{1}{|c|}{ Type } & \multicolumn{1}{c|}{ Attribute Description } \\
\hline ExternalIdentifier & $0 . *^{*}$ & ExternalIdentifier & $\begin{array}{l}\text { For associating an ID with the } \\
\text { election. }\end{array}$ \\
\hline Name & $0 . .1$ & String & $\begin{array}{l}\text { For including a name for the } \\
\text { election; the name could be the } \\
\text { same name as appears on the ballot. }\end{array}$ \\
\hline StartDate & 1 & date & The first day of the election. \\
\hline EndDate & $0 . .1$ & date & $\begin{array}{l}\text { For an election that spans multiple } \\
\text { days, the last day of the election. }\end{array}$ \\
\hline
\end{tabular}




\subsection{Class ElectionAdministration}

Used in response messages; ElectionAdministration optionally includes ContactMethod to specify contact information for the election authority.

ElectionAdministration

-Name : String [0..1]

-Uri : anyURI [0..*]

Figure 13 - ElectionAdministration

\begin{tabular}{|l|c|c|l|}
\hline \multicolumn{1}{|c|}{ Attribute } & Multiplicity & Type & \multicolumn{1}{c|}{ Attribute Description } \\
\hline \{ContactMethod\} & $0 . .^{*}$ & ContactMethod & For including various contact information. \\
\hline \{Location\} & $0 . .1$ & Location & Location of the election authority. \\
\hline Name & $0 . .1$ & String & Name of the election authority. \\
\hline Uri & $0 . .^{*}$ & anyURI & A URL for the election authority. \\
\hline
\end{tabular}




\subsection{Class ElectionBasedBallotRequest}

Used in request messages as an implementation of BallotRequest in which a ballot for a single election event is requested.

ElectionBasedBallotRequest

Figure 14 - ElectionBasedRequest

\begin{tabular}{|l|c|c|c|}
\hline \multicolumn{1}{|c|}{ Attribute } & Multiplicity & Type & Attribute Description \\
\hline EElection\} & 1 & Election & The election for which the ballot is requested. \\
\hline
\end{tabular}




\subsection{Class Error}

Used in response messages; RequestRejection includes this class to describe the errors that caused the rejection.

\begin{tabular}{|c|}
\hline \multicolumn{1}{|c|}{ Error } \\
\hline attnibutes \\
-Name : RequestError [1] \\
-OtherError: String [0..1] \\
-Ref : String [0..1] \\
\hline \hline
\end{tabular}

Figure 15 - Error

\begin{tabular}{|l|c|c|l|}
\hline \multicolumn{1}{|c|}{ Attribute } & Multiplicity & \multicolumn{1}{c|}{ Type } & \multicolumn{1}{c|}{ Attribute Description } \\
\hline Name & 1 & RequestError & Used to indicate the type of error. \\
\hline OtherError & $0 . .1$ & String & Used when Name value is other. \\
\hline Ref & $0 . .1$ & String & $\begin{array}{l}\text { Reference (e.g. Xpath) to the entity that the } \\
\text { error applies. }\end{array}$ \\
\hline
\end{tabular}




\subsection{Class Externalldentifier}

Used in request and response messages by BallotStyle, Election, Party and ReportingUnit, which optionally include this class for associating a jurisdiction's codes, i.e., identifiers, with political parties or geopolitical units such as counties, towns, precincts, etc. Multiple occurrences of ExternalIdentifier can be used to associate multiple codes, e.g., if there is a desire to associate multiple codes with an object such as state-specific codes as well as OCD-IDs (Open Civic Data Identifiers) [11].

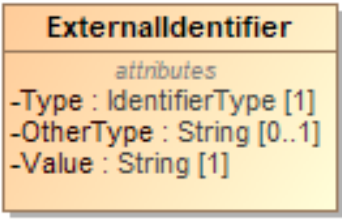

Figure 16 - Externalldentifier

\begin{tabular}{|l|c|c|l|}
\hline \multicolumn{1}{|c|}{ Attribute } & Multiplicity & \multicolumn{1}{c|}{ Type } & \multicolumn{1}{c|}{ Attribute Description } \\
\hline Type & 1 & IdentifierType & An identifier type, e.g., FIPS. \\
\hline OtherType & $0 . .1$ & String & Used when Type value is other. \\
\hline Value & 1 & String & The identifier used by the jurisdiction. \\
\hline
\end{tabular}




\subsection{Class File}

Used in request and response messages; VoterId optionally uses this class for FileValue to specify a filename for voter identification purposes such as for a utility bill. AdditionalInfo also optionally includes FileValue.

File extends the xsd:base64Binary type to add the attributes for filename and (Multi-Purpose Internet Mail Extensions) MIME type, e.g., application/pdf for a file of type PDF.

The Image element uses this class as a base class, thus Image can be used when the type of file is for an image, e.g., image/png.

\begin{tabular}{|l|}
\multicolumn{1}{|c|}{ File } \\
\hline \multicolumn{1}{c|}{ atthibutes } \\
«simpleContentw-Data : base64Binary [1] \\
-FileName : String [0..1] \\
-MimeType : String [0..1]
\end{tabular}

Figure 17 - File

\begin{tabular}{|l|c|c|l|}
\hline \multicolumn{1}{|c|}{ Attribute } & Multiplicity & \multicolumn{1}{c|}{ Type } & \multicolumn{1}{c|}{ Attribute Description } \\
\hline Data & 1 & base64Binary & The file content encoded using base64. \\
\hline FileName & $0 . .1$ & String & The filename. \\
\hline MimeType & $0 . .1$ & String & The MIME type associated with the file. \\
\hline
\end{tabular}




\subsection{Class Image}

Used in request and response messages; Signature optionally includes this class to indicate that a file contains an image of a voter's signature. Image uses File as a base class, thus attributes of File can be included in Image.

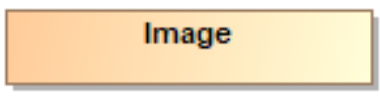

Figure 18 - Image 


\subsection{Class LatLng}

Used in response messages by Location, which optionally includes this element to specify the latitude and longitude of a voter's voting location.

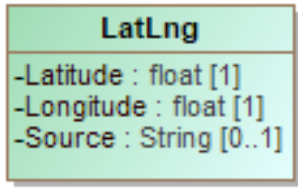

Figure 19 - LatLng

\begin{tabular}{|l|c|c|l|}
\hline \multicolumn{1}{|c|}{ Attribute } & Multiplicity & Type & \multicolumn{1}{c|}{ Attribute Description } \\
\hline Latitude & 1 & float & Latitude of the location. \\
\hline Longitude & 1 & float & Longitude of the location. \\
\hline Source & $0 . .1$ & String & $\begin{array}{l}\text { System used to perform the lookup from } \\
\text { location name to lat/lng, e.g., the name of a } \\
\text { geocoding service. }\end{array}$ \\
\hline
\end{tabular}




\subsection{Class Location}

Used in response messages by ElectionAdministration and ReportingUnit, which optionally include this element to specify the address and directions to a voter's voting location. The LatLng element can be included to specify the latitude and longitude of the voting location.

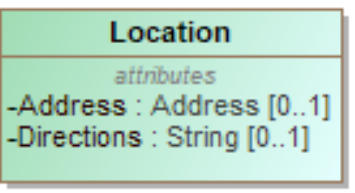

Figure 20 - Location

\begin{tabular}{|l|c|c|l|}
\hline \multicolumn{1}{|c|}{ Attribute } & Multiplicity & Type & \multicolumn{1}{c|}{ Attribute Description } \\
\hline Address & $0 . .1$ & Address & Address of the voting location. \\
\hline Directions & $0 . .1$ & String & Directions to the voting location. \\
\hline \{LatLng & $0 . .1$ & LatLng & Latitude/longitude of the voting location. \\
\hline
\end{tabular}




\subsection{Class Name}

Used in request and response messages; Voter includes this class for specifying the name of a voter and, optionally, for specifying a previous name of the voter, using PreviousName instead of Name. RequestHelper also includes this class for specifying the name of a request helper.

Multiple occurrences of the MiddleName attribute can be used as needed, e.g., for names with additional middle names or nicknames such as "John Andrew Winston (Jack) Smith".

All elements are optional, however at least FullName must be included if the other attributes are not.

\begin{tabular}{|l|}
\multicolumn{1}{|c|}{ Name } \\
\hline \multicolumn{1}{|c|}{ attributes } \\
-FirstName : String [0..1] \\
-FullName : String [0..1] \\
-LastName : String [0..1] \\
-MiddleName : String [0..* -Mordered\} \\
-Prefix: String [0..1] \\
-Suffix : String [0..1] \\
\hline \hline
\end{tabular}

Figure 21 - Name

\begin{tabular}{|l|c|c|l|}
\hline \multicolumn{1}{|c|}{ Attribute } & Multiplicity & \multicolumn{1}{c|}{ Type } & \multicolumn{1}{c|}{ Attribute Description } \\
\hline FirstName & $0 . .1$ & String & Person's first (given) name. \\
\hline FullName & $0 . .1$ & String & Person's full name. \\
\hline LastName & $0 . .1$ & String & Person's last (family) name. \\
\hline MiddleName & $0 .{ }^{*}$ & String & Person's middle name. \\
\hline Prefix & $0 . .1$ & String & A prefix associated with the person, e.g., Mr. \\
\hline Suffix & $0 . .1$ & String & A suffix associated with the person, e.g., Jr. \\
\hline
\end{tabular}




\subsection{Class Party}

Used in request and response messages by BallotStyle, which optionally includes this type to specify the associated political party such as for closed primaries, and by Voter, which optionally includes this type to specify a voter's political party.

\begin{tabular}{|c|}
\hline Party \\
\hline attributes \\
-Abbreviation : String [0..1] \\
-Name : String [1]
\end{tabular}

Figure 22 - Party

\begin{tabular}{|l|c|c|l|}
\hline \multicolumn{1}{|c|}{ Attribute } & $\begin{array}{c}\text { Multiplic } \\
\text { ity }\end{array}$ & \multicolumn{1}{|c|}{ Type } & \multicolumn{1}{|c|}{ Attribute Description } \\
\hline Abbreviation & $0 . .1$ & String & $\begin{array}{l}\text { Short name for the party, e.g., } \\
\text { "DEM". }\end{array}$ \\
\hline EExternalIdentifier $\}$ & $0 . .^{*}$ & ExternalIdentifier & For associating an ID with the party. \\
\hline Name & 1 & String & $\begin{array}{l}\text { Official full name of the party, e.g., } \\
\text { "Republican". }\end{array}$ \\
\hline
\end{tabular}




\subsection{Class PermanentBallotRequest}

Used in request messages as a subtype of BallotRequest, which serves to request ballots for election events that the voter is qualified on a long term basis. Although "permanent", the request may be subject to renewal or cancellation procedures.

PermanentBallotRequest

Figure 23 - PermanentBallotRequest 


\subsection{Class PhoneContactMethod}

Used in request and response messages; RequestHelper, and RequestProxy use this class to specify a telephone number as well as the capabilities of the telephone, e.g., sms, fax, etc.

PhoneContactMethod is subtype of ContactMethod. Thus, the elements that include ContactMethod could use PhoneContactMethod as applicable.

\begin{tabular}{|c|}
\hline PhoneContactMethod \\
\hline -Capability : PhoneCapability [0..* $]$ \\
\hline
\end{tabular}

Figure 24 - PhoneContactMethod

\begin{tabular}{|c|c|c|l|}
\hline Attribute & Multiplicity & \multicolumn{1}{|c|}{ Type } & \multicolumn{1}{|c|}{ Attribute Description } \\
\hline Capability & $0 . *^{*}$ & PhoneCapability & $\begin{array}{l}\text { Specifies the phone's capabilities, e.g., fax, } \\
\text { sms. }\end{array}$ \\
\hline
\end{tabular}




\subsection{Class ReportingUnit}

Used in response messages; RequestSuccess and VoterRecord include this class so as to provide a list of geopolitical geography associated with the voter's registration, e.g., the voter's precinct, polling place, districts, etc. VoterParticipation optionally includes this class to specify the polling place used by the voter. The Type attribute uses the ReportingUnitType enumeration to specify the type of geopolitical geography being defined. If the reporting unit type is not listed in enumeration ReportingUnitType, use "other" and include the reporting unit type (that is not listed in the enumeration) in OtherType.

The IsDistricted boolean is not strictly necessary, as it is possible to identify districts by their Type attribute. However, if the type of district is not listed in the ReportingUnitType enumeration and therefore OtherType is used, then IsDistricted is necessary. The IsDistricted boolean can also be used to signify that a ReportingUnit defined as a jurisdiction, e.g., a county, is also used as a district for, e.g., county-wide contests.

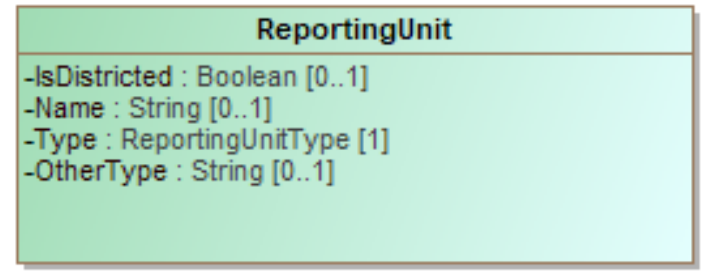

Figure 25 - ReportingUnit

\begin{tabular}{|l|c|c|l|}
\hline \multicolumn{1}{|c|}{ Attribute } & Multiplicity & \multicolumn{1}{|c|}{ Type } & \multicolumn{1}{c|}{ Attribute Description } \\
\{ExternalIdentifier\} & $0 . *^{*}$ & ExternalIdentifier & $\begin{array}{l}\text { For associating an ID with the } \\
\text { ReportingUnit. }\end{array}$ \\
\hline IsDistricted & $0 . .1$ & Boolean & $\begin{array}{l}\text { Boolean to indicate that the } \\
\text { reporting unit is a district. }\end{array}$ \\
\hline \{Location\} & $0 . .1$ & $\underline{\text { Location }}$ & Location of the district office. \\
\hline Name & $0 . .1$ & String & Name of the reporting unit. \\
\hline Type & 1 & ReportingUnitType & $\begin{array}{l}\text { Enumerated type of reporting } \\
\text { unit, e.g., district, precinct. }\end{array}$ \\
\hline OtherType & $0 . .1$ & String & Used when Type value is other. \\
\hline
\end{tabular}




\subsection{Class RequestAcknowledgement}

Used in response messages for indicating that the request was received but action on the request is pending.

RequestAcknowledgement

Figure 26 - RequestAcknowledgement 


\subsection{Class RequestHelper}

Used in request messages; VoterRecordsRequest optionally includes this element to specify information about a request helper, i.e., a request assistant or witness involved in a voter's request.

RequestHelper optionally includes the Name element to specify the registration helper's name and optionally includes the Signature element if a registration helper's signature is required.

\begin{tabular}{|c|}
\hline \multicolumn{1}{|c|}{ RequestHelper } \\
\hline attributes \\
-Address : Address [0..1] \\
-Phone : PhoneContactMethod [0..1] \\
-Type : VoterHelperType [1] \\
\hline \hline
\end{tabular}

Figure 27 - RequestHelper

\begin{tabular}{|l|c|c|l|}
\hline \multicolumn{1}{|c|}{ Attribute } & Multiplicity & \multicolumn{1}{c|}{ Type } & \multicolumn{1}{c|}{ Attribute Description } \\
\hline Address & $0 . .1$ & Address & Address of the request helper. \\
\hline$\{$ Name $\}$ & $0 . .1$ & $\underline{\text { Name }}$ & To specify the name of the helper. \\
\hline Phone & $0 . .1$ & $\underline{\text { PhoneContactMethod }}$ & Request helper's phone number. \\
\hline$\{$ Signature $\}$ & $0 . .1$ & $\underline{\text { Signature }}$ & To specify the signature of the helper. \\
\hline Type & 1 & VoterHelperType & $\begin{array}{l}\text { To specify the type of helper, e.g., } \\
\text { assistant. }\end{array}$ \\
\hline
\end{tabular}




\subsection{Class RequestProxy}

Used in request messages; VoterRecordsRequest optionally includes this class to specify information about a request proxy involved in a voter records request. OriginTransactionId can be used to include an optional identifier of the originating external transaction from the proxy, e.g., used for the transaction ID generated by a MVA application enacting a voter registration request to a registration portal application (on behalf of a citizen obtaining a driver's license).

This sub-element is not to be confused with TransactionId in VoterRecordsRequest, which is used to include a transaction ID of the voter records request, e.g., the transaction ID of the registration portal's voter records request.

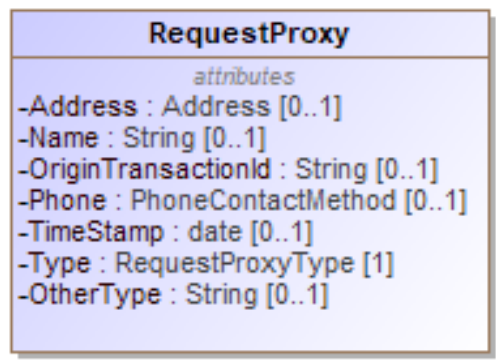

Figure 28 - RequestProxy

\begin{tabular}{|l|c|c|l|}
\hline \multicolumn{1}{|c|}{ Attribute } & Multiplicity & \multicolumn{1}{|c|}{ Type } & \multicolumn{1}{|c|}{ Attribute Description } \\
\hline Address & $0 . .1$ & Address & $\begin{array}{l}\text { An address associated with the } \\
\text { proxy. }\end{array}$ \\
\hline Name & 0.1 & String & $\begin{array}{l}\text { A name associated with the } \\
\text { proxy. }\end{array}$ \\
\hline OriginTransactionId & $0 . .1$ & String & $\begin{array}{l}\text { An identifier associated with the } \\
\text { transaction between the proxy } \\
\text { and, e.g., the registration portal. }\end{array}$ \\
\hline Phone & $0 . .1$ & PhoneContactMethod & $\begin{array}{l}\text { A phone number associated with } \\
\text { the proxy. }\end{array}$ \\
\hline TimeStamp & 0.1 & date & $\begin{array}{l}\text { The date of the request from the } \\
\text { proxy. }\end{array}$ \\
\hline Type & 1 & RequestProxyType & $\begin{array}{l}\text { The type of the requesting proxy, } \\
\text { e.g., motor-vehicle-office, voter- } \\
\text { via-email. }\end{array}$ \\
\hline OtherType & $0 . .1$ & String & $\begin{array}{l}\text { Used when OtherType value is } \\
\text { other. }\end{array}$ \\
\hline
\end{tabular}




\subsection{Class RequestRejection}

Used in response messages for indicating that the request failed. The Error attribute is used to indicate the type of error that occurred. The AdditionalDetails attribute can be used to provide more information as to the rejection.

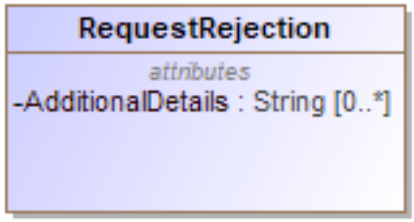

Figure 29 - RequestRejection

\begin{tabular}{|l|c|c|l|}
\hline \multicolumn{1}{|c|}{ Attribute } & Multiplicity & Type & \multicolumn{1}{|c|}{ Attribute Description } \\
\hline AdditionalDetails & $0 . .^{*}$ & String & $\begin{array}{l}\text { Used to provide additional details as } \\
\text { applicable. }\end{array}$ \\
\hline$\{$ Error\} & $0 . .^{*}$ & Error & $\begin{array}{l}\text { For associating a RequestRejection with one or } \\
\text { more Error. }\end{array}$ \\
\hline
\end{tabular}




\subsection{Class RequestSuccess}

Used in response messages for indicating a successful response to a request. The Action attribute is used to indicate the action that occurred, which may differ from what was requested. For example, a request for a new voter registration may succeed, but if the voter was already registered, the response may indicate a registration update as opposed to a registration create.

The response also includes, optionally, other information useful to the voter, including a description of the voter's polling place, districts (i.e., contests) associated with the polling place, or other geopolitical geographies such as the voter's precinct.

\begin{tabular}{|c|}
\hline \multicolumn{1}{|c|}{ RequestSuccess } \\
\hline attributes \\
-Action : SuccessAction [0..* $\left.{ }^{-1}\right]$ \\
-EffectiveDate : date [0..1] \\
\hline \hline
\end{tabular}

Figure 30 - RequestSuccess

\begin{tabular}{|l|c|c|l|}
\hline \multicolumn{1}{|c|}{ Attribute } & Multiplicity & \multicolumn{1}{|c|}{ Type } & \multicolumn{1}{c|}{$\begin{array}{c}\text { Attribute } \\
\text { Description }\end{array}$} \\
\hline Action & $0 . *^{*}$ & SuccessAction & $\begin{array}{l}\text { Used to indicate the } \\
\text { action that occurred. }\end{array}$ \\
\hline \{District\} & $0 . *^{*}$ & Reportingunit & $\begin{array}{l}\text { One or more districts } \\
\text { associated with the } \\
\text { voter's precinct. }\end{array}$ \\
\hline EffectiveDate & $0 . .1$ & date & $\begin{array}{l}\text { The effective date of } \\
\text { the action. }\end{array}$ \\
\hline$\{$ ElectionAdministration\} & $0 . .1$ & ElectionAdministration & $\begin{array}{l}\text { The election } \\
\text { administration that } \\
\text { conducts elections for } \\
\text { the voter. }\end{array}$ \\
\hline$\{$ Locality & $0 .{ }^{*}$ & Reportingunit & $\begin{array}{l}\text { Other geographies such } \\
\text { as the voter's precinct. }\end{array}$ \\
\hline$\{$ PollingPlace\} & 0.1 & ReportingUnit & $\begin{array}{l}\text { The voter's polling } \\
\text { place. }\end{array}$ \\
\hline
\end{tabular}




\subsection{Class Signature}

Used in request and response messages; Voter optionally includes this class for specifying information about a voter's signature on a registration request. If there is a need to include

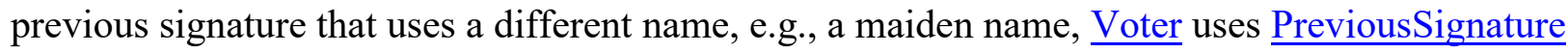
instead of Signature.

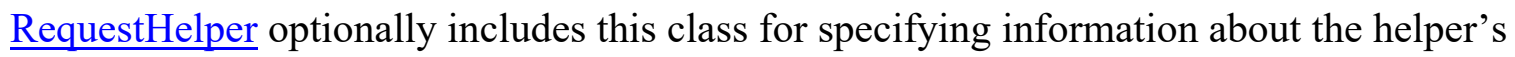
signature.

Source is used to specify the source of the voter's signature, for example, on file at a motor vehicles administration. FileValue is used to include an image of the voter's signature.

\begin{tabular}{|l|}
\hline \multicolumn{1}{|c|}{ Signature } \\
\hline \multicolumn{1}{|c|}{ attributes } \\
-Date : date [0..1] \\
-FileValue : Image [0..1] \\
-Source : SignatureSource [0..1] \\
-OtherSource : String [0..1] \\
-Type: SignatureType [0..1] \\
-OtherType : String [0..1] \\
\hline
\end{tabular}

Figure 31 - Signature

\begin{tabular}{|l|c|c|l|}
\hline \multicolumn{1}{|c|}{ Attribute } & Multiplicity & \multicolumn{1}{c|}{ Type } & \multicolumn{1}{c|}{ Attribute Description } \\
\hline Date & $0 . .1$ & date & $\begin{array}{l}\text { The date of the signature, i.e., when } \\
\text { created. }\end{array}$ \\
\hline FileValue & $0 . .1$ & Image & The signature image in base 64 binary. \\
\hline Source & $0 . .1$ & $\underline{\text { SignatureSource }}$ & A source for the signature, e.g., MVA. \\
\hline OtherSource & $0 . .1$ & String & Used when $\underline{\text { Source value is other. }}$ \\
\hline Type & $0 . .1$ & $\underline{\text { SignatureType }}$ & A signature type, e.g., dynamic. \\
\hline OtherType & $0 . .1$ & String & Used when $\underline{\text { Type value is other. }}$ \\
\hline
\end{tabular}




\subsection{Class TemporalBallotRequest}

Used in request messages as a subtype of BallotRequest in which election opportunities for which the voter is qualified during a given time frame may be requested.

\begin{tabular}{|l|}
\hline TemporalBallotRequest \\
\hline attributes \\
-StartDate : date [1] \\
-EndDate : date [1] \\
\hline \hline
\end{tabular}

Figure 32 - TemporalBallotRequest

\begin{tabular}{|l|c|c|l|}
\hline \multicolumn{1}{|c|}{ Attribute } & Multiplicity & Type & \multicolumn{1}{|c|}{ Attribute Description } \\
\hline StartDate & 1 & date & The date at which the request comes into effect. \\
\hline EndDate & 1 & date & $\begin{array}{l}\text { The date at which the request is no longer } \\
\text { effective. }\end{array}$ \\
\hline
\end{tabular}




\subsection{Class Voter}

Used in request and response messages to contain attributes specific to identifying a voter.

\begin{tabular}{|l|}
\hline \multicolumn{1}{|c|}{ Voter } \\
\hline \multicolumn{1}{|c|}{ attributes } \\
-DateOfBirth : date [0..1] \\
-Ethnicity : String [0..1] \\
-Gender : String [0..1] \\
-MailingAddress : Address [0..1] \\
-PreviousResidenceAddress : Address [0..1] \\
-ResidenceAddress : Address [1] \\
-ResidenceAddresslsMailingAddress : Boolean [0..1] \\
\hline
\end{tabular}

Figure $33-$ Voter

\begin{tabular}{|c|c|c|c|}
\hline Attribute & Multiplicity & Type & Attribute Description \\
\hline$\{$ ContactMethod $\}$ & $0 .{ }^{*}$ & ContactMethod & $\begin{array}{l}\text { How to contact the voter, listed } \\
\text { in order of preference. }\end{array}$ \\
\hline DateofBirth & $0 . .1$ & date & $\begin{array}{l}\text { The voter's data of birth in } \\
\text { YYYY-MM-DD } \\
\text { format. }\end{array}$ \\
\hline Ethnicity & $0 . .1$ & String & The voter's ethnicity. \\
\hline Gender & $0 . .1$ & String & $\begin{array}{l}\text { Older systems may not } \\
\text { understand values other than } \\
\text { 'Male' or 'Female' (the only } \\
\text { choices available on FPCA). }\end{array}$ \\
\hline MailingAddress & $0 . .1$ & Address & $\begin{array}{l}\text { Where the voter receives postal } \\
\text { mail, mapped to the FGDC } \\
\text { specification Address classes. }\end{array}$ \\
\hline$\{$ Name $\}$ & 1 & $\underline{\text { Name }}$ & Voter's name. \\
\hline$\{$ Party $\}$ & $0 . .1$ & Party & Voter's political party. \\
\hline$\{$ PreviousName $\}$ & $0 . .1$ & $\underline{\text { Name }}$ & A voter's previous name. \\
\hline PreviousResidenceAddress & $0 . .1$ & Address & $\begin{array}{l}\text { Where the voter was previously } \\
\text { registered, mapped to the } \\
\text { FGDC specification Address } \\
\text { classes. }\end{array}$ \\
\hline$\{$ PreviousSignature $\}$ & $0 . .1$ & $\underline{\text { Signature }}$ & $\begin{array}{l}\text { Information about a previous } \\
\text { voter signature on the } \\
\text { registration form. }\end{array}$ \\
\hline
\end{tabular}




\begin{tabular}{|c|c|c|c|}
\hline Attribute & Multiplicity & Type & Attribute Description \\
\hline ResidenceAddress & 1 & Address & $\begin{array}{l}\text { Where the voter is registered or } \\
\text { requests to be registered, } \\
\text { mapped to the FGDC } \\
\text { specification Address classes. }\end{array}$ \\
\hline $\begin{array}{l}\text { ResidenceAddressIsMailing } \\
\text { Address }\end{array}$ & $0 . .1$ & Boolean & $\begin{array}{l}\text { If set to true, MailingAddress } \\
\text { need not be included. }\end{array}$ \\
\hline$\{$ Signature $\}$ & $0 . .1$ & $\underline{\text { Signature }}$ & $\begin{array}{l}\text { Information about the voter } \\
\text { signature on the registration } \\
\text { form. }\end{array}$ \\
\hline$\{$ VoterClassification $\}$ & $0 . *^{*}$ & $\frac{\text { VoterClassifica }}{\text { tion }}$ & $\begin{array}{l}\text { How the voter is classified per } \\
\text { assertions the voter has made } \\
\text { on a registration form. }\end{array}$ \\
\hline$\{$ VoterId $\}$ & $0 . .^{*}$ & $\underline{\text { VoterId }}$ & $\begin{array}{l}\text { Information to provide voter } \\
\text { identity. }\end{array}$ \\
\hline
\end{tabular}




\subsection{Class VoterClassification}

Used in request and response messages; Voter optionally includes this class to describe a voter's classification per criteria on the voter's request form, e.g., united-states-citizen or eighteen-onelection-day.

VoterClassification includes assertions of the voter in response to the voter request form criteria. For example, an assertion of true may be used with a criterion of united-states-citizen. Assertions can be negative, such as providing an assertion of false for a criterion of felon, an assertion of unknown to indicate that the voter does not know whether they meet or do not meet the specific criteria on the form or an assertion of other, in which the assertion is specified by the value of OtherAssertion.

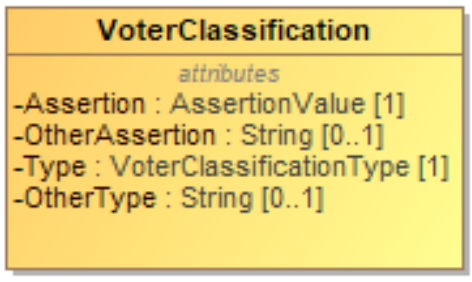

Figure $34-$ VoterClassification

\begin{tabular}{|l|c|c|l|}
\hline \multicolumn{1}{|c|}{ Attribute } & Multiplicity & \multicolumn{1}{c|}{ Type } & \multicolumn{1}{c|}{ Attribute Description } \\
\hline Assertion & 1 & AssertionValue & $\begin{array}{l}\text { A positive, negative, other or } \\
\text { unknown assertion }\end{array}$ \\
\hline OtherAssertion & $0 . .1$ & String & A locally defined assertion value. \\
\hline Type & 1 & VoterClassificationType & A classification type, e.g., felon. \\
\hline OtherType & $0 . .1$ & String & Used when Type value is other. \\
\hline
\end{tabular}




\subsection{Class Voterld}

Used in request and response messages to include information about a voter's identification that may be required in a registration request. Voter includes VoterId.

AttestNoSuchId is used to attest that the voter has no ID of a specified type, thus it must be included with a value of true if attesting that the voter has no ID for that specified type. It can be included with a value of false to attest that the voter does have an ID of the specified type, in which case either StringValue or FileValue must be included; however, it is assumed to be false if not included.

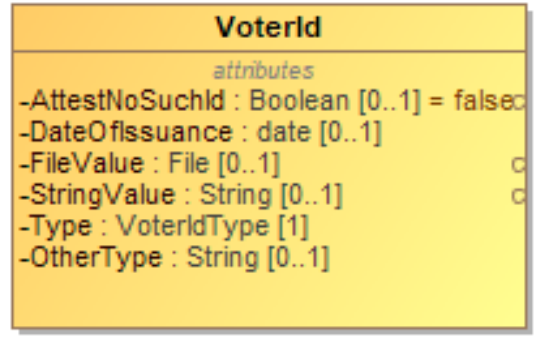

Figure $35-$ Voterld

\begin{tabular}{|l|c|c|l|}
\hline \multicolumn{1}{|c|}{ Attribute } & Multiplicity & Type & \multicolumn{1}{c|}{ Attribute Description } \\
\hline AttestNoSuchId & $0 . .1$ & Boolean & $\begin{array}{l}\text { Used to attest that the voter has no ID. } \\
\text { Assumed to be false if not present. }\end{array}$ \\
\hline DateOfIssuance & $0 . .1$ & date & Date the ID was issued. \\
\hline FileValue & $0 . .1$ & $\underline{\text { File }}$ & Used to include a file name for the ID. \\
\hline StringValue & $0 . .1$ & String & Used to include the ID as a string. \\
\hline Type & 1 & $\underline{\text { voterIdType }}$ & The type of voter ID. \\
\hline OtherType & $0 . .1$ & String & Used when Type value is other. \\
\hline
\end{tabular}




\subsection{Class VoterParticipation}

Used in response messages for indicating an election that the voter participated in. Participation does not imply a counted ballot.

VoterParticipation

Figure 36 - VoterParticipation

\begin{tabular}{|l|c|c|l|}
\hline \multicolumn{1}{|c|}{ Attribute } & Multiplicity & Type & \multicolumn{1}{c|}{ Attribute Description } \\
\hline BBallotstyle\} & $0 . .1$ & Ballotstyle & $\begin{array}{l}\text { For associating the voter participation to a } \\
\text { specific ballot style, such to a partisan } \\
\text { ballot in a closed primary. }\end{array}$ \\
\hline$\{$ Election\} & 1 & Election & $\begin{array}{l}\text { For associating the voter participation to a } \\
\text { specific election event. }\end{array}$ \\
\hline \{PollingLocation\} & $0 . .1$ & $\underline{\text { ReportingUnit }}$ & The polling place used by the voter. \\
\hline
\end{tabular}




\subsection{Class VoterRecord}

Used in response messages to represent a voter record stored in a Voter Registration Database (VRDB). VoterRecord optionally contains additional information useful to the voter, including a description of the voter's polling place, districts associated with the voter's precinct, or other geopolitical geographies such as the voter's precinct.

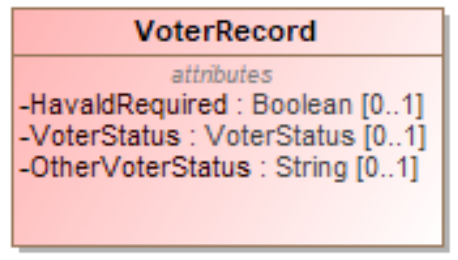

Figure 37 - VoterRecord

\begin{tabular}{|c|c|c|c|}
\hline Attribute & Multiplicity & Type & Attribute Description \\
\hline \{District $\}$ & $0 . .^{*}$ & ReportingUnit & $\begin{array}{l}\text { One or more districts associated } \\
\text { with the voter's precinct. }\end{array}$ \\
\hline $\begin{array}{l}\text { \{ElectionAdministrat } \\
\text { ion\} }\end{array}$ & $0 . .1$ & $\frac{\text { ElectionAdministra }}{\text { tion }}$ & $\begin{array}{l}\text { The election administration that } \\
\text { conducts elections for the voter. }\end{array}$ \\
\hline HavaIdRequired & $0 . .1$ & Boolean & $\begin{array}{l}\text { Indicates that the voter must } \\
\text { present identification at the } \\
\text { polls per HAVA (Help America } \\
\text { Vote Act of 2002). }\end{array}$ \\
\hline$\{$ Locality $\}$ & $0 .{ }^{*}$ & ReportingUnit & $\begin{array}{l}\text { Other geographies such as the } \\
\text { voter's precinct. }\end{array}$ \\
\hline \{PollingLocation\} & $0 . .1$ & ReportingUnit & The voter's polling place. \\
\hline$\{$ Voter $\}$ & 1 & Voter & $\begin{array}{l}\text { For details specific to a } \\
\text { particular voter. }\end{array}$ \\
\hline$\{$ VoterParticipation $\}$ & $0 .{ }^{*}$ & VoterParticipation & $\begin{array}{l}\text { For associating a VoterRecord to } \\
\text { elections the voter has } \\
\text { participated in. }\end{array}$ \\
\hline Voterstatus & $0 . .1$ & Voterstatus & $\begin{array}{l}\text { The status of the VoterRecord, } \\
\text { possibly to indicate the ability } \\
\text { to receive a regular ballot. }\end{array}$ \\
\hline OtherVoterStatus & $0 . .1$ & String & $\begin{array}{l}\text { Used when VoterStatus value is } \\
\text { other. }\end{array}$ \\
\hline
\end{tabular}




\subsection{Class VoterRecordResults}

Used in response messages for indicating a successful response to a lookup request.

A lookup for a single voter may result in multiple VoterRecord objects being returned. This can occur if the voter has duplicate records in the VRDB, or if the criteria specified in the lookup request was broad.

VoterRecordResults

Figure 38 - VoterRecordResults

\begin{tabular}{|c|c|c|c|}
\hline Attribute & Multiplicity & Type & \multicolumn{1}{c|}{ Attribute Description } \\
\hline \{VoterRecord $\}$ & $0 . .^{*}$ & VoterRecord & The voter record(s) returned. \\
\hline
\end{tabular}




\subsection{Class VoterRecordsRequest}

The root element for request messages, for defining items pertaining to the status and type of the voter records request and when it was generated. VoterRecordsRequest includes the Subject association to specify various information about the voter in question. It includes the BallotRequest association to handle a request for a ballot; this request may be part of an FPCA form registration or may be submitted independently.

\begin{tabular}{|c|}
\hline $\begin{array}{c}\text { «Root» } \\
\text { VoterRecordsRequest }\end{array}$ \\
\hline $\begin{array}{l}\text { aitinbutes } \\
\text {-Form : RequestForm [0..1] } \\
\text {-OtherForm : String [0..1] } \\
\text {-GeneratedDate : date [1] } \\
\text {-lssuer : String [0..1] } \\
\text { RequestMethod : RequestMethod [1] } \\
\text {-OtherRequestMethod : String [0..1] } \\
\text {-SelectedLanguage : language [0..1] } \\
\text {-Transactionld : String [0..1] } \\
\text {-Type : VoterRequestType [1...] } \\
\text {-OtherType : String [0..1] } \\
\text {-VendorApplicationld : String [0..1] }\end{array}$ \\
\hline
\end{tabular}

Figure 39 - VoterRecordsRequest

\begin{tabular}{|l|c|c|l|}
\hline \multicolumn{1}{|c|}{ Attribute } & $\begin{array}{c}\text { Multiplici } \\
\text { ty }\end{array}$ & \multicolumn{1}{|c|}{ Type } & \multicolumn{1}{|c|}{ Attribute Description } \\
\hline \{AdditionalInfo\} & $0 . *^{*}$ & AdditionalInfo & $\begin{array}{l}\text { For including other information not } \\
\text { specified by this model. }\end{array}$ \\
\hline$\{$ BallotRequest $\}$ & $0 . .1$ & $\underline{\text { BallotRequest }}$ & $\begin{array}{l}\text { Specifies information relating to a } \\
\text { request for a ballot. }\end{array}$ \\
\hline Form & $0 . .1$ & $\underline{\text { RequestForm }}$ & $\begin{array}{l}\text { If the request is for a voter registration, } \\
\text { the registration form used by the voter. }\end{array}$ \\
\hline OtherForm & $0 . .1$ & String & Used when Form value is other. \\
\hline GeneratedDate & 1 & date & $\begin{array}{l}\text { The date that the voter records request } \\
\text { was generated. }\end{array}$ \\
\hline Issuer & $0 . .1$ & $\begin{array}{l}\text { The name of the issuer of the voter } \\
\text { records request transaction, e.g., State } \\
\text { of West Virginia Voter Registration } \\
\text { Portal. }\end{array}$ \\
\hline SRequestHelper $\}$ & $0 . *^{*}$ & $\underline{\text { RequestHelper }}$ & $\begin{array}{l}\text { Included if the registration involves a } \\
\text { registration assistant organization. }\end{array}$ \\
\hline RequestMethod & 1 & $\underline{\text { RequestMethod }}$ & $\begin{array}{l}\text { The method used by the voter to } \\
\text { register. }\end{array}$ \\
\hline
\end{tabular}




\begin{tabular}{|l|c|c|l|}
\hline \multicolumn{1}{|c|}{ Attribute } & $\begin{array}{c}\text { Multiplici } \\
\text { ty }\end{array}$ & \multicolumn{1}{|c|}{ Type } & \multicolumn{1}{|c|}{ Attribute Description } \\
\hline OtherRequestMethod & $0 . .1$ & String & $\begin{array}{l}\text { Used when RequestMethod value is } \\
\text { other. }\end{array}$ \\
\hline \{RequestProxy\} & $0 . .1$ & RequestProxy & $\begin{array}{l}\text { Included if the registration request is } \\
\text { via a proxy, e.g., the MVA. }\end{array}$ \\
\hline SelectedLanguage & $0 . .1$ & language & $\begin{array}{l}\text { The language specified by the voter, if } \\
\text { any. }\end{array}$ \\
\hline \{Subject\} & 1 & Voter & $\begin{array}{l}\text { Specifies information about the voter } \\
\text { who is the subject of the request. }\end{array}$ \\
\hline TransactionId & $0 . .1$ & String & $\begin{array}{l}\text { An identifier of the voter records } \\
\text { request transaction. }\end{array}$ \\
\hline Type & $1 . . *$ & VoterRequestType & The type of request, e.g., registration. \\
\hline OtherType & $0 . .1$ & String & Used when RequestType value is other. \\
\hline VendorApplicationId & $0 . .1$ & String & $\begin{array}{l}\text { An identifier of the vendor application } \\
\text { generating the voter registration } \\
\text { request, e.g., X-VRDB Version 3.1.a. }\end{array}$ \\
\hline
\end{tabular}




\subsection{Class VoterRecordsResponse}

The root element for response messages, for defining items pertaining to the status of a response to a voter records request. VoterRecordsResponse is an abstract class with four subtypes that get used according to the type of response:

- $\quad$ RequestAcknowledgement, used to indicate an acknowledgement only.

- $\quad$ VoterRecordResults, used to provide a set of voter records.

- $\quad$ RequestRejection, used to indicate a failure and the type of failure.

- $\quad$ RequestSuccess, used to indication that a successful action occurred and the type of action, which may differ from the type of action requested.

VoterRecordsResponse optionally includes the TransactionId attribute associated with the voter records request.

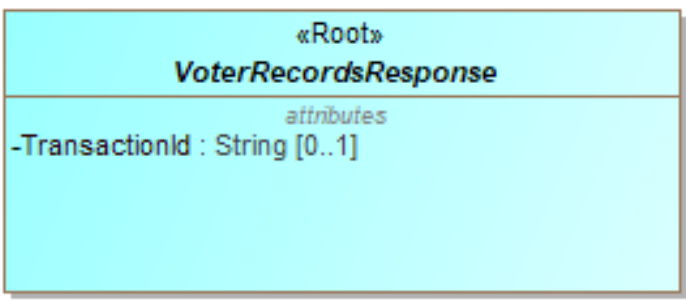

Figure 40 - VoterRecordsResponse

\begin{tabular}{|c|c|c|l|}
\hline Attribute & Multiplicity & Type & \multicolumn{1}{|c|}{ Attribute Description } \\
\hline TransactionId & $0 . .1$ & String & $\begin{array}{l}\text { Transaction ID associated with the voter } \\
\text { records request. }\end{array}$ \\
\hline
\end{tabular}




\subsection{Enumeration AssertionValue}

Enumeration for assertions from a voter or a third party, such as a motor vehicles administration (MVA) in response to questions on a registration form, used in the Assertion attribute of VoterClassification. Used in request and response messages.

\begin{tabular}{|l|}
\hline \multicolumn{1}{|c|}{$\begin{array}{c}\text { Aenumeration: } \\
\text { AssertionValue }\end{array}$} \\
\hline enumeration literals \\
no \\
yes \\
unknown \\
other \\
\hline
\end{tabular}

Figure 41 - AssertionValue

\begin{tabular}{|l|l|}
\hline \multicolumn{1}{|c|}{ Value } & \multicolumn{1}{c|}{ Value Description } \\
\hline no & For a voter's or third party's assertion of "no" or "false". \\
\hline yes & For a voter's or third party's assertion of "yes" or "true". \\
\hline unknown & For a voter's or third party's assertion of "unknown". \\
\hline other & For a voter's or third party's assertion of "other". \\
\hline
\end{tabular}




\subsection{Enumeration BallotReceiptMethod}

Enumeration for methods for delivering a ballot to the voter, used in the BallotReceiptPreference attribute of BallotRequest. The sub-element may be repeated multiple times with different values as applicable, e.g., to specify both mail and online. Used in request messages.

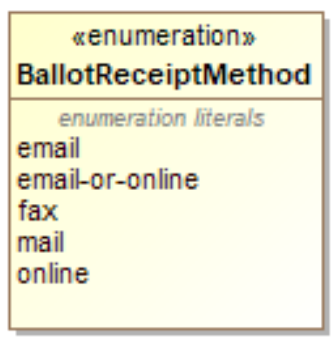

Figure 42 - BallotReceiptMethod

\begin{tabular}{|l|l|}
\hline \multicolumn{1}{|c|}{ Value } & \multicolumn{1}{c|}{ Value Description } \\
\hline email & For email only. \\
\hline email-or-online & $\begin{array}{l}\text { For electronic mail or downloadable from a website (this value is } \\
\text { ambiguous, thus the separate values for email and online). }\end{array}$ \\
\hline fax & For use of a fax. \\
\hline mail & For postal mail. \\
\hline online & $\begin{array}{l}\text { For downloadable from a website, e.g., the voter is sent a hypertext link } \\
\text { to a ballot. }\end{array}$ \\
\hline
\end{tabular}




\subsection{Enumeration ContactMethodType}

Enumeration for methods for contacting a voter or an election administration office, used in the

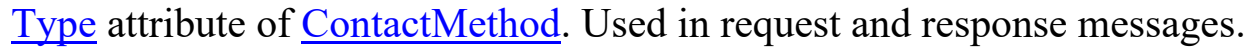

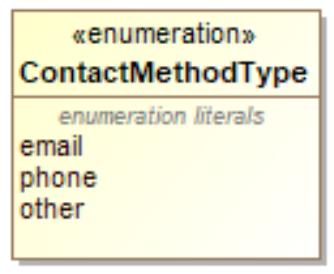

Figure 43 - ContactMethodType

\begin{tabular}{|l|l|}
\hline \multicolumn{1}{|c|}{ Value } & \multicolumn{1}{c|}{ Value Description } \\
\hline email & For electronic mail. \\
\hline phone & For use of a phone. \\
\hline other & $\begin{array}{l}\text { Used when the type of contact method is not included in this } \\
\text { enumeration. }\end{array}$ \\
\hline
\end{tabular}




\subsection{Enumeration IdentifierType}

Enumeration for election data-related codes in the ExternalIdentifier class. Used in request and response messages.

\begin{tabular}{|l|}
\hline $\begin{array}{l}\text { «enumeration» } \\
\text { IdentifierType }\end{array}$ \\
\hline fips \\
local-level \\
national-level \\
ocd-id \\
state-level \\
other
\end{tabular}

Figure 44 - IdentifierType

\begin{tabular}{|l|l|}
\hline \multicolumn{1}{|c|}{ Value } & \multicolumn{1}{c|}{ Value Description } \\
\hline fips & For FIPS codes. \\
\hline local-level & For a code that is specific to a county or other similar locality. \\
\hline national-level & For a code that is used at the national level other than ocd-id. \\
\hline ocd-id & For Open Civic Data identifiers. \\
\hline state-level & For a code that is specific to a state. \\
\hline other & Used when the type of code is not included in this enumeration. \\
\hline
\end{tabular}




\subsection{Enumeration PhoneCapability}

Enumeration for telephone capabilities, used in the Capability attribute of PhoneContactMethod. Used in request and response messages.

\begin{tabular}{l} 
wenumeration: \\
PhoneCapability \\
\hline enumeration literals \\
fax \\
mms \\
sms \\
voice
\end{tabular}

Figure 45 - PhoneCapability

\begin{tabular}{|l|l|}
\hline \multicolumn{1}{|c|}{ Value } & \multicolumn{1}{c|}{ Value Description } \\
\hline fax & For telephones that include facsimile capabilities. \\
\hline mms & $\begin{array}{l}\text { For telephones that contain Multimedia Messaging Service (MMS) } \\
\text { capabilities. }\end{array}$ \\
\hline sms & $\begin{array}{l}\text { For telephones that contain Short Messaging Service (SMS) } \\
\text { capabilities. }\end{array}$ \\
\hline voice & For telephones that contain voice capabilities. \\
\hline
\end{tabular}




\subsection{Enumeration ReportingUnitType}

Enumeration for the type of geopolitical unit, used in the Type sub-element in the ReportingUnit element. Used in request and response messages.

\begin{tabular}{|l|}
\hline \multicolumn{1}{|c|}{ wenumeration. } \\
ReportingUnitType \\
\hline ballot-batch \\
ballot-style-area \\
borough \\
city \\
city-council \\
combined-precinct \\
congressional \\
county \\
county-council \\
drop-box \\
judicial \\
municipality \\
polling-place \\
precinct \\
school \\
special \\
split-precinct \\
state \\
state-house \\
state-senate \\
town \\
township \\
utility \\
village \\
vote-center \\
ward \\
water \\
other \\
\\
\hline \hline
\end{tabular}

Figure 46 - ReportingUnitType

\begin{tabular}{|l|l|}
\hline \multicolumn{1}{|c|}{ Value } & \multicolumn{1}{c|}{ Value Description } \\
\hline ballot-batch & $\begin{array}{l}\text { Used for reporting batches of ballots that may cross precinct } \\
\text { boundaries. }\end{array}$ \\
\hline ballot-style-area & Used for ballot style areas generally composed of precincts. \\
\hline borough & $\begin{array}{l}\text { Used in CT, NJ, PA, other states, and New York City for boroughs. For } \\
\text { AK and LA, see county. }\end{array}$ \\
\hline city & $\begin{array}{l}\text { Used for a city that reports results and/or for the district that } \\
\text { encompasses it. }\end{array}$ \\
\hline city-council & Used for city council districts. \\
\hline
\end{tabular}




\begin{tabular}{|c|c|}
\hline Value & Value Description \\
\hline combined-precinct & $\begin{array}{l}\text { Used for one or more precincts that have been combined for the } \\
\text { purposes of reporting. Used for "Ward" if "Ward" is used } \\
\text { interchangeably with "CombinedPrecinct". }\end{array}$ \\
\hline congressional & Used for U.S. Congressional districts. \\
\hline county & $\begin{array}{l}\text { Used for a county and/or for the district that encompasses it. In AK, } \\
\text { used for counties that are called boroughs. In LA, used for parishes. }\end{array}$ \\
\hline county-council & Used for county council districts. \\
\hline drop-box & Used for a dropbox for ballots. \\
\hline judicial & Used for judicial districts. \\
\hline municipality & $\begin{array}{l}\text { Used as applicable for various units such as towns, townships, villages } \\
\text { that report votes and/or for the district that encompasses it. }\end{array}$ \\
\hline polling-place & Used for a polling place. \\
\hline precinct & $\begin{array}{l}\text { Used also for "Ward" or "District" when these terms are used } \\
\text { interchangeably with "Precinct". }\end{array}$ \\
\hline school & Used for a school district. \\
\hline special & Used for a special district. \\
\hline split-precinct & Used for splits of precincts. \\
\hline state & Used for a state and/or for the district that encompasses it. \\
\hline state-house & Used for a state house or assembly district. \\
\hline state-senate & Used for a state senate district. \\
\hline town & $\begin{array}{l}\text { Used in some New England states as a type of municipality that reports } \\
\text { votes and/or for the district that encompasses it. }\end{array}$ \\
\hline township & $\begin{array}{l}\text { Used in some mid-western states as a type of municipality that reports } \\
\text { votes and/or for the district that encompasses it. }\end{array}$ \\
\hline utility & Used for a utility district. \\
\hline village & $\begin{array}{l}\text { Used as a type of municipality that reports votes and/or for the district } \\
\text { that encompasses it. }\end{array}$ \\
\hline vote-center & Used for a vote center. \\
\hline ward & Used for combinations or groupings of precincts or other units. \\
\hline water & Used for a water district. \\
\hline other & Used for other types of reporting units not included in this enumeration. \\
\hline
\end{tabular}




\subsection{Enumeration RequestError}

Enumeration for registration-related errors, used in the Name attribute of RequestRejection. Used in response messages.

\begin{tabular}{|l|}
\hline \multicolumn{1}{|c|}{ wenumeration» } \\
RequestError \\
\hline $\begin{array}{l}\text { enumeration literals } \\
\text { identity-lookup-failed } \\
\text { incomplete } \\
\text { ineligible } \\
\text { invalid-form } \\
\text { other }\end{array}$ \\
\hline \hline
\end{tabular}

Figure 47 - RequestError

\begin{tabular}{|l|l|}
\hline \multicolumn{1}{|c|}{ Value } & \multicolumn{1}{c|}{ Value Description } \\
\hline identity-lookup-failed & A lookup on the voter's identity failed. \\
\hline incomplete & The registration request is incomplete. \\
\hline ineligible & The voter is ineligible to be registered. \\
\hline invalid-form & The registration form specified is invalid. \\
\hline other & Used when the type of error is not included in this enumeration. \\
\hline
\end{tabular}




\subsection{Enumeration RequestForm}

Enumeration for types of registration forms, used in the Form attribute of VoterRecordsRequest. Used in request messages.

\begin{tabular}{|l|}
\hline $\begin{array}{c}\text { «enumeration» } \\
\text { RequestForm }\end{array}$ \\
\hline enumeration literals \\
fpca \\
nvra \\
other
\end{tabular}

Figure 48 - RequestForm

\begin{tabular}{|l|l|}
\hline \multicolumn{1}{|c|}{ Value } & \multicolumn{1}{c|}{ Value Description } \\
\hline fpca & For the Federal Post Card Application form. \\
\hline nvra & For the National Voter Registration Act form. \\
\hline other & Used when the type of form is not included in this enumeration. \\
\hline
\end{tabular}




\subsection{Enumeration RequestMethod}

Enumeration for the method used by the voter to register, used in the RequestMethod attribute of VoterRecordsRequest. Used in request messages.

\begin{tabular}{|c|}
\hline $\begin{array}{c}\text { «enumeration» } \\
\text { RequestMethod }\end{array}$ \\
\hline $\begin{array}{l}\text { enumeration literals } \\
\text { armed-forces-recruitment-office } \\
\text { motor-vehicle-office } \\
\text { other-agency-designated-by-state } \\
\text { public-assistance-office } \\
\text { registration-drive-from-advocacy-group-or-political-party } \\
\text { state-funded-agency-serving-persons-with-disabilities } \\
\text { voter-via-election-registrars-office } \\
\text { voter-via-email } \\
\text { voter-via-fax } \\
\text { voter-via-internet } \\
\text { voter-via-mail } \\
\text { unknown } \\
\text { other }\end{array}$ \\
\hline
\end{tabular}

Figure 49 - RequestMethod

\begin{tabular}{|l|l|}
\hline \multicolumn{1}{|c|}{ Value } & \multicolumn{1}{c|}{ Value Description } \\
\hline $\begin{array}{l}\text { armed-forces-recruitment- } \\
\text { office }\end{array}$ & The voter assisted by an armed forces recruitment office. \\
\hline motor-vehicle-office & The voter via an MVA. \\
\hline $\begin{array}{l}\text { other-agency-designated- } \\
\text { by-state }\end{array}$ & The voter assisted by an unspecified state-designated agency. \\
\hline public-assistance-office & The voter assisted by a public assistance office. \\
\hline $\begin{array}{l}\text { registration-drive-from- } \\
\text { advocacy-group-or- } \\
\text { political-party }\end{array}$ & The voter via a registration drive. \\
\hline $\begin{array}{l}\text { state-funded-agency- } \\
\text { serving-persons-with- } \\
\text { disabilities }\end{array}$ & $\begin{array}{l}\text { The voter assisted by a state-designated agency serving persons with } \\
\text { disabilities. }\end{array}$ \\
\hline $\begin{array}{l}\text { voter-via-election- } \\
\text { registrars-office }\end{array}$ & The voter via an election or registrar's office. \\
\hline voter-via-email & The voter via email. \\
\hline voter-via-fax & The voter via fax. \\
\hline voter-via-internet & The voter via the Internet, e.g., a website. \\
\hline voter-via-mail & The voter via postal mail. \\
\hline unknown & The method used is unknown. \\
\hline
\end{tabular}




\subsection{Enumeration RequestProxyType}

Enumeration for the registration proxy, e.g., the MVA, involved in the voter's registration request, used in the Type attribute of RequestProxy. Used in request messages.

\begin{tabular}{|l|}
\hline \multicolumn{1}{|c|}{$\begin{array}{l}\text { «enumeration: } \\
\text { RequestProxyType }\end{array}$} \\
\hline \multicolumn{1}{|c|}{ enumeration literals } \\
armed-forces-recruitment-office \\
motor-vehicle-office \\
other-agency-designated-by-state \\
public-assistance-office \\
registration-drive-from-advocacy-group-or-political-party \\
state-funded-agency-serving-persons-with-disabilities \\
other
\end{tabular}

Figure 50 - RequestProxyType

\begin{tabular}{|l|l|}
\hline \multicolumn{1}{|c|}{ Value } & \multicolumn{1}{c|}{ Value Description } \\
\hline $\begin{array}{l}\text { armed-forces-recruitment- } \\
\text { office }\end{array}$ & The voter assisted by an armed forces recruitment office. \\
\hline motor-vehicle-office & The voter via an MVA. \\
\hline $\begin{array}{l}\text { other-agency-designated- } \\
\text { by-state }\end{array}$ & The voter assisted by an unspecified state-designated agency. \\
\hline public-assistance-office & The voter assisted by a public assistance office. \\
\hline $\begin{array}{l}\text { registration-drive-from- } \\
\text { advocacy-group-or- } \\
\text { political-party }\end{array}$ & The voter via a registration drive. \\
\hline $\begin{array}{l}\text { state-funded-agency- } \\
\text { serving-persons-with- } \\
\text { disabilities }\end{array}$ & $\begin{array}{l}\text { The voter assisted by a state-designated agency serving persons with } \\
\text { disabilities. }\end{array}$ \\
\hline other & Used when the type of source is not included in this enumeration. \\
\hline
\end{tabular}




\subsection{Enumeration SignatureSource}

Enumeration for source of the voter's signature, used in the Source sub-element of Signature. Used in request and response messages.

\begin{tabular}{|l|}
\hline \multicolumn{1}{|c|}{ «enumeration: } \\
Signature Source \\
\hline enumeration literals \\
dmv \\
local \\
state \\
voter \\
other \\
\hline
\end{tabular}

Figure 51 - SignatureSource

\begin{tabular}{|l|l|}
\hline \multicolumn{1}{|c|}{ Value } & \multicolumn{1}{c|}{ Value Description } \\
\hline dmv & For the department of motor vehicles or motor vehicle administration. \\
\hline local & For an unspecified local source. \\
\hline state & For an unspecified state source. \\
\hline voter & The voter has included a signature on the form. \\
\hline other & $\begin{array}{l}\text { Used when the source of the signature is not included in this } \\
\text { enumeration. }\end{array}$ \\
\hline
\end{tabular}




\subsection{Enumeration SignatureType}

Enumeration for the type of voter signature, used in the Type sub-element of Signature. Used in request and response messages.

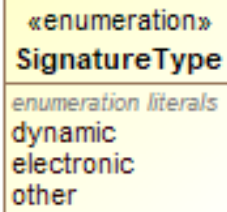

Figure 52 - SignatureType

\begin{tabular}{|l|l|}
\hline \multicolumn{1}{|c|}{ Value } & \multicolumn{1}{c|}{ Value Description } \\
\hline dynamic & $\begin{array}{l}\text { For use with biometrics or other artifacts captured as part of the act of } \\
\text { the voter signing the registration form. }\end{array}$ \\
\hline electronic & $\begin{array}{l}\text { For a facsimile of the signature applied to a marking surface, e.g., } \\
\text { paper. }\end{array}$ \\
\hline other & Used when the type of signature is not included in this enumeration. \\
\hline
\end{tabular}




\subsection{Enumeration SuccessAction}

Enumeration for a response to a voter records request, indicating that the response to the request is successful and the action that occurred, used in the Action sub-element of RequestSuccess. The success action may not necessarily match the requested action. Used in response messages.

\begin{tabular}{|l|}
\hline \multicolumn{1}{|c|}{ «enumerations } \\
SuccessAction \\
\hline \multicolumn{1}{|c|}{ enumeration literals } \\
address-updated \\
name-updated \\
registration-cancelled \\
registration-created \\
registration-updated \\
status-updated \\
other
\end{tabular}

Figure 53 - SuccessAction

\begin{tabular}{|l|l|}
\hline \multicolumn{1}{|c|}{ Value } & \multicolumn{1}{c|}{ Value Description } \\
\hline address-updated & For indicating that an address was updated. \\
\hline name-updated & For indicating that a name was updated. \\
\hline registration-cancelled & For indicating that a registration was cancelled. \\
\hline registration-created & For indicating that a registration was created. \\
\hline registration-updated & For indicating that a registration was updated. \\
\hline status-updated & For indicating that a registration status was updated. \\
\hline other & $\begin{array}{l}\text { Used for other types of success actions not included in this } \\
\text { enumeration. }\end{array}$ \\
\hline
\end{tabular}




\subsection{Enumeration VoterClassificationType}

Enumeration for voter status classifications, used in the Type attribute of VoterClassification. Whether the voter status, e.g., eighteen-on-election-day, is true, false, or unknown depends on the value of the Assertion attribute. Used in request and response messages.

\begin{tabular}{|l|}
\hline \multicolumn{1}{|c|}{ «enumeration» } \\
VoterClassificationType \\
\hline \multicolumn{1}{|c|}{ enumeration literals } \\
activated-national-guard \\
active-duty \\
active-duty-spouse-or-dependent \\
citizen-abroad-intent-to-return \\
citizen-abroad-return-uncertain \\
citizen-abroad-never-resided \\
deceased \\
declared-incompetent \\
eighteen-on-election-day \\
felon \\
permanently-denied \\
protected-voter \\
restored-felon \\
united-states-citizen \\
other \\
\hline
\end{tabular}

Figure 54 - VoterClassificationType

\begin{tabular}{|l|l|}
\hline \multicolumn{1}{|c|}{ Value } & \multicolumn{1}{c|}{ Value Description } \\
\hline activated-national-guard & $\begin{array}{l}\text { The voter is an activated National Guard member on State orders } \\
\text { (FPCA). }\end{array}$ \\
\hline active-duty & $\begin{array}{l}\text { The voter is a member of the Uniformed Services or Merchant Marine } \\
\text { on active duty (FPCA). }\end{array}$ \\
\hline $\begin{array}{l}\text { active-duty-spouse-or- } \\
\text { dependent }\end{array}$ & The voter is an eligible spouse or dependent (FPCA). \\
\hline $\begin{array}{l}\text { citizen-abroad-intent-to- } \\
\text { return }\end{array}$ & $\begin{array}{l}\text { The voter is a US citizen residing outside the US and has intention to } \\
\text { return (FPCA). }\end{array}$ \\
\hline $\begin{array}{l}\text { citizen-abroad-return- } \\
\text { uncertain }\end{array}$ & $\begin{array}{l}\text { The voter is a US citizen residing outside the US and their return is } \\
\text { uncertain (FPCA). }\end{array}$ \\
\hline $\begin{array}{l}\text { citizen-abroad-never- } \\
\text { resided }\end{array}$ & The voter is a US citizen and has never resided in the US (FPCA). \\
\hline deceased & The voter is deceased (NVRA). \\
\hline declared-incompetent & The voter has been declared incompetent (NVRA). \\
\hline eighteen-on-election-day & The voter will be 18 on election day (NVRA). \\
\hline felon & The voter is a felon (NVRA). \\
\hline
\end{tabular}




\begin{tabular}{|l|l|}
\hline \multicolumn{1}{|c|}{ Value } & \multicolumn{1}{c|}{ Value Description } \\
\hline permanently-denied & The voter has not been permanently denied (NVRA). \\
\hline protected-voter & The voter status is protected (NVRA). \\
\hline restored-felon & The voter is a restored felon (NVRA). \\
\hline united-states-citizen & The voter is a United States citizen (NVRA). \\
\hline other & $\begin{array}{l}\text { Used when the type of voter classification is not included in this } \\
\text { enumeration. }\end{array}$ \\
\hline
\end{tabular}




\subsection{Enumeration VoterHelperType}

Enumeration for types of registration helpers, used in the Type attribute of RequestHelper. Used in request messages.

\begin{tabular}{l} 
«enumeration* \\
VoterHelperType \\
\hline enumeration literals \\
assistant \\
witness
\end{tabular}

Figure 55 - VoterHelperType

\begin{tabular}{|l|l|}
\hline \multicolumn{1}{|c|}{ Value } & \multicolumn{1}{c|}{ Value Description } \\
\hline assistant & For a registration assistant. \\
\hline witness & For a registration witness. \\
\hline
\end{tabular}




\subsection{Enumeration VoterldType}

Enumeration for the type of voter ID, used in the Type attribute of VoterId. Used in request and response messages.

\begin{tabular}{|l||}
\hline \multicolumn{1}{|c|}{\begin{tabular}{c|} 
"enumeration» \\
VoterldType
\end{tabular}} \\
\hline drivers-license \\
local-voter-registration-id litera/s \\
ssn \\
ssn4 \\
state-id \\
state-voter-registration-id \\
unspecified-document \\
unspecified-document-with-name-and-address \\
unspecified-document-with-photo-identification \\
unknown \\
other \\
\hline \hline
\end{tabular}

Figure 56 - VoterldType

\begin{tabular}{|l|l|}
\hline \multicolumn{1}{|c|}{ Value } & \multicolumn{1}{c|}{ Value Description } \\
\hline drivers-license & Used for a driver's license. \\
\hline $\begin{array}{l}\text { local-voter-registration- } \\
\text { id }\end{array}$ & Used for a local voter registration record ID. \\
\hline ssn & Used for a complete Social Security number. \\
\hline ssn4 & Used for the last four digits of a Social Security number. \\
\hline state-id & Used for a state ID that is not a state voter registration ID. \\
\hline $\begin{array}{l}\text { state-voter-registration- } \\
\text { id }\end{array}$ & Used for a state's voter registration record ID. \\
\hline $\begin{array}{l}\text { unspecified-document } \\
\text { unspecified-document- } \\
\text { with-name-and-address }\end{array}$ & $\begin{array}{l}\text { Used for an unspecified document, not known whether the document } \\
\text { contains name, address, or photo ID. }\end{array}$ \\
\hline $\begin{array}{l}\text { unspecified-document- } \\
\text { with-photo-identification } \\
\text { as a utility bill. }\end{array}$ & Used for a document that contains a photograph of the voter. \\
\hline unknown & Used for documentation that was not captured. \\
\hline other & Used when the type of ID is not included in this enumeration. \\
\hline
\end{tabular}




\subsection{Enumeration VoterRequestType}

Enumeration for the type of voter records request, used in the Type attribute of VoterRecordsRequest. Used in request messages.

\begin{tabular}{|l|}
\hline \multicolumn{1}{|c|}{ «enumeration» } \\
VoterRequestType \\
\hline \multicolumn{1}{|c|}{ enumeration literals } \\
ballot-request \\
lookup \\
registration \\
other
\end{tabular}

Figure 57 - VoterRequestType

\begin{tabular}{|l|l|}
\hline \multicolumn{1}{|c|}{ Value } & \multicolumn{1}{c|}{ Value Description } \\
\hline ballot-request & $\begin{array}{l}\text { For requesting a ballot, possibly in conjunction with an FPCA } \\
\text { registration request. }\end{array}$ \\
\hline lookup & For a voter registration lookup. \\
\hline registration & For a voter registration request. \\
\hline other & Used when the type of request is not included in this enumeration. \\
\hline
\end{tabular}




\subsection{Enumeration VoterStatus}

Enumeration for the status of the voter in a voter registration database, used in the VoterStatus attribute of the VoterRecord. Used in response messages.

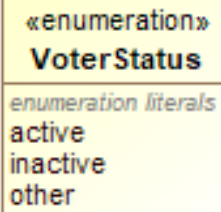

Figure $58-$ VoterStatus

\begin{tabular}{|l|l|}
\hline \multicolumn{1}{|c|}{ Value } & \multicolumn{1}{c|}{ Value Description } \\
\hline active & For a voter in active status. \\
\hline inactive & For a voter in inactive status. \\
\hline other & Used when the type of voter status is not included in this enumeration. \\
\hline
\end{tabular}




\section{$4 \quad$ XML and JSON Usage Examples}

This section contains several examples showing voter records request and responses in XML and JSON, all using the NVRA form. The examples are:

- Voter Registration Request - XML

- Voter Registration Request - JSON

- Voter Registration Response - XML

In the voter records request examples, note that a significant majority of the statements deal with specifying addresses using the FGDC standard [6].

\subsection{Example 1: NVRA-style Voter Registration Request in XML}

Figure 59 shows a fictitious digital NVRA-style registration request for "Jackie Nichole Davidson" in the State of Ohio using XML. This request is for an address update, and an example of the filled-out NVRA-style form is shown.

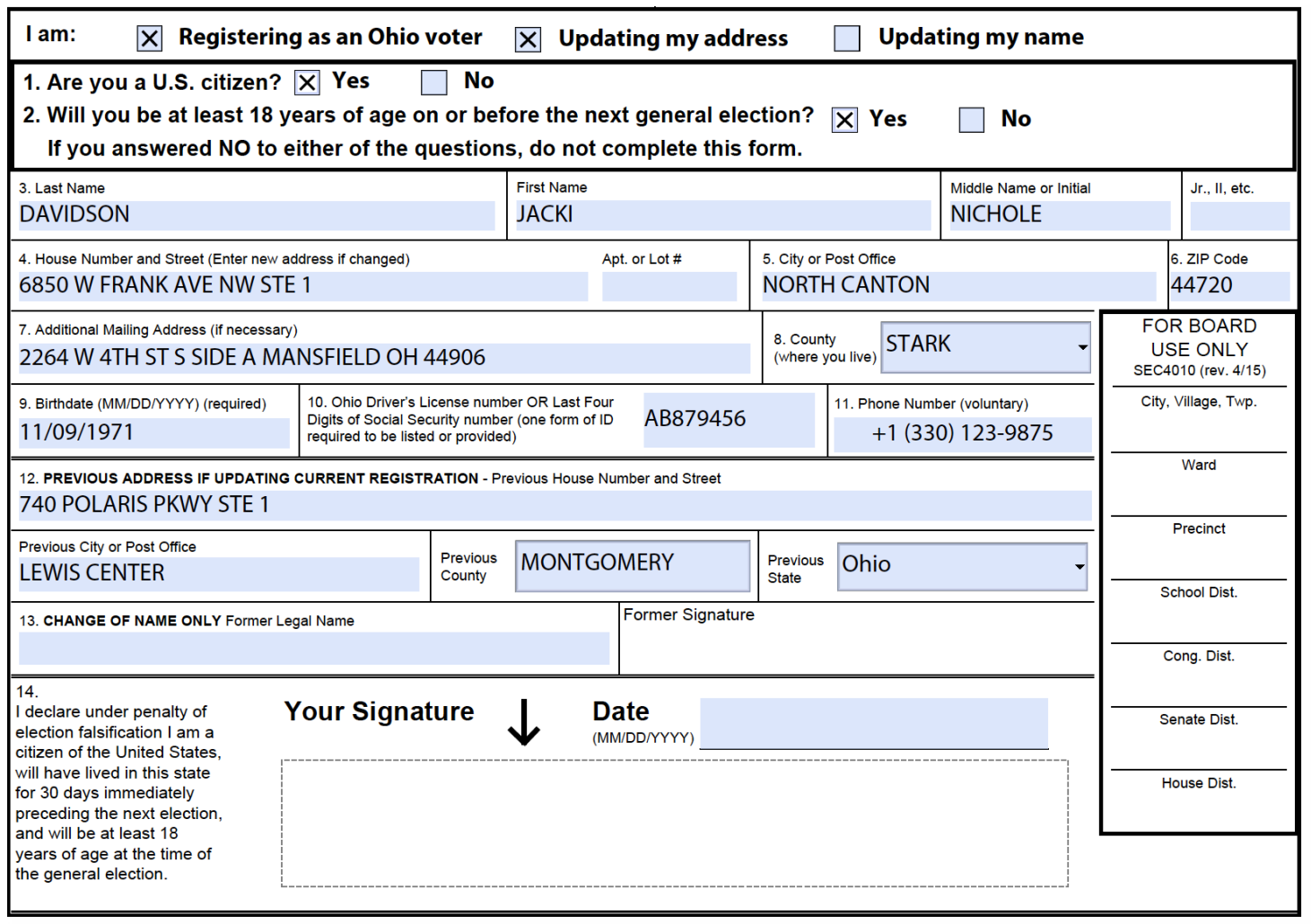

Figure 59 - Example NVRA-style form for a voter address update request 
The XML for the voter registration request that contains the information exported from the form is shown below. Note that in lines 7 through 10 the <Additional Info > element is being used to indicate that the voter's language preference is English; this is needed because the XML (and JSON) schema does not include a voter-preferred language element but the application at the portal required it. Thus, <Additionalinfo $\rangle$ can be used to add other elements that are required by the application but not present in the schema. 


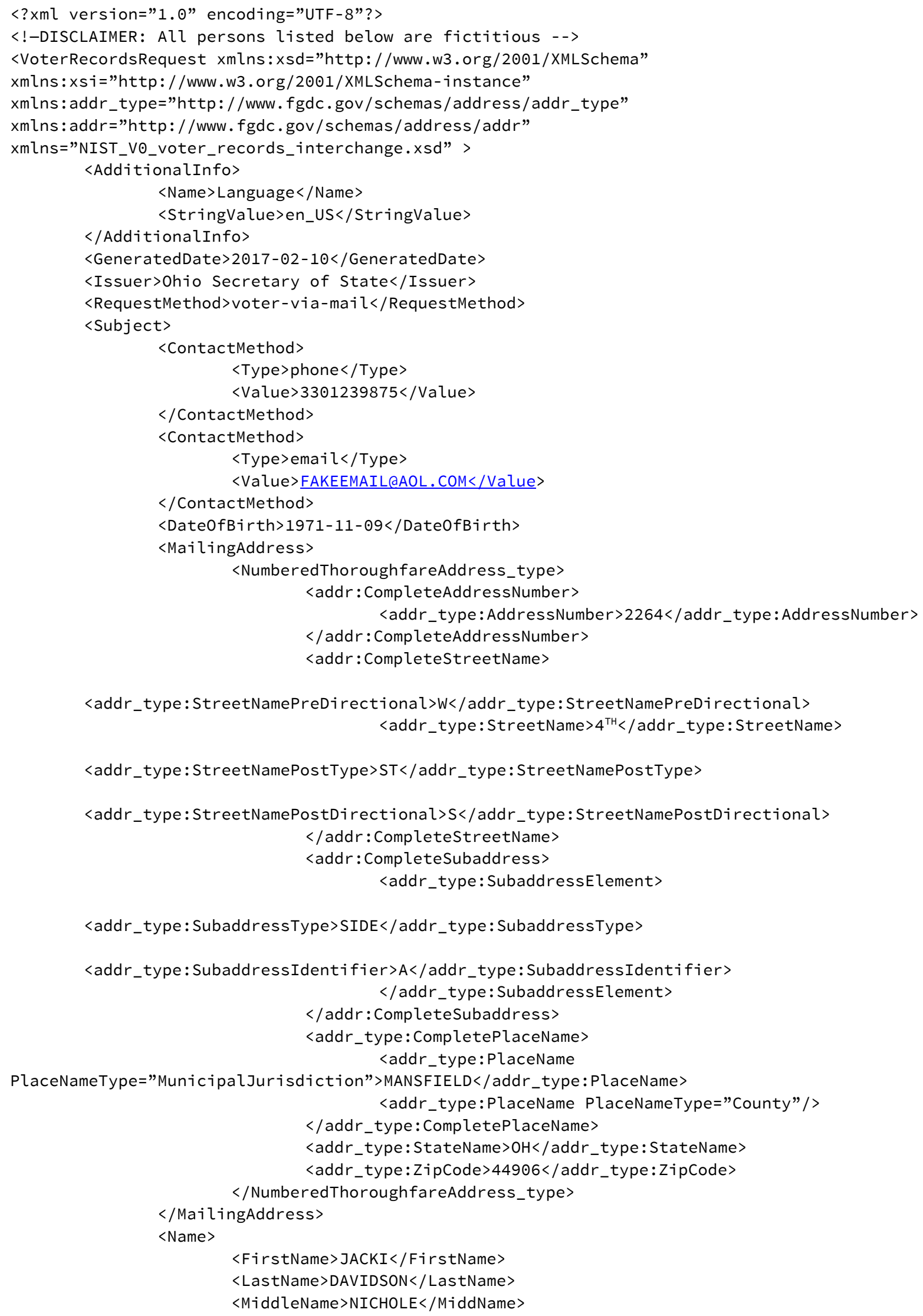


60

61

62

63

64

65

66

67

68

69

70

71

72

73

74

75

76

77

78

79

80

81

82

83

84

85

86

87

88

89

90

91

92

93

94

95

96

97

98

99

100

101

102

103

104

105

106

107

108

109

110

111

112

113

114

115

116

117

118
$</$ Name $>$

<PreviousResidenceAddress>

<NumberedThoroughfareAddress_type>

<addr:CompleteAddressNumber>

<addr_type: AddressNumber $>740</$ addr_type: AddressNumber >

$</$ addr: CompleteAddressNumber >

<addr:CompleteStreetName>

<addr_type:StreetNamePreDirectional/>

<addr_type:StreetName>POLARIS</addr_type:StreetName>

<addr_type:StreetNamePostType>PKWY</addr_type:StreetNamePostType>

<addr_type:StreetNamePostDirectional/>

$</$ addr: CompleteStreetName>

<addr:CompleteSubaddress>

<addr_type: SubaddressElement>

<addr_type: SubaddressType>STE</addr_type: SubaddressType>

<addr_type: SubaddressIdentifier $>1</ a d d r_{-}$type: SubaddressIdentifier >

$</$ addr_type:SubaddressElement $>$

$</$ addr: CompleteSubaddress $>$

<addr_type:CompletePlaceName>

<addr_type:PlaceName

PlaceNameType="MunicipalJurisdiction" $>$ LEWIS CENTER $</$ addr_type: $P$ laceName $>$ <addr_type: PlaceName PlaceNameType="County" />

$</$ addr_type:CompletePlaceName $>$

$<$ addr_type:StateName $>\mathrm{OH}</$ addr_type: StateName>

<addr_type:ZipCode>43035</addr_type:ZipCode>

$</$ NumberedThoroughfareAddress_type $>$

$</$ PreviousResidenceAddress $>$

$<$ ResidenceAddress $>$

<NumberedThoroughfareAddress_type>

<addr:CompleteAddressNumber >

<addr_type: AddressNumber $>6850</$ addr_type: AddressNumber >

$</$ addr: CompleteAddressNumber >

<addr:CompleteStreetName>

<addr_type:StreetNamePreDirectional $>W</ a d d r_{\text {_type }}$ StreetNamePreDirectional>

<addr_type:StreetName>FRANK</addr_type:StreetName>

<addr_type:StreetNamePostType>AVE</addr_type:StreetNamePostType>

<addr_type:StreetNamePostDirectional >NW</addr_type:StreetNamePostDirectional>

$</$ addr: CompleteStreetName>

<addr:CompleteSubaddress>

<addr_type:SubaddressElement>

<addr_type: SubaddressType>STE</addr_type: SubaddressType>

<addr_type: SubaddressIdentifier >1</addr_type:SubaddressIdentifier> $</$ addr_type:SubaddressElement $>$

$</$ addr: CompleteSubaddress $>$

$<$ addr_type:CompletePlaceName>

<addr_type:PlaceName

PlaceNameType="MunicipalJurisdiction" $>$ NORTH CANTON $</$ addr_type: $P$ laceName $>$ <addr_type:PlaceName PlaceNameType="County" $>$ STARK

$</ a d d r_{-}$type:PlaceName $>$

$</$ addr_type:CompletePlaceName $>$

$<$ addr_type: StateName $>\mathrm{OH}</$ addr_type: StateName> 
119

120

121

122

123

124

125

126

127

128

129

130

131

132

133

134

135

136

137

138

139

140

141

142

143

144

145

146

147

148

149

150

151

152

153
<addr_type:ZipCode $>44720</$ addr_type:ZipCode>

$</$ NumberedThoroughfareAddress_type $>$

$\langle/$ ResidenceAddress $>$

$<$ VoterClassification $>$

<Assertion>yes</Assertion>

$<$ Type >eighteen-on-election-day</Type $>$

$</$ VoterClassification $>$

$<$ VoterClassification $>$

<Assertion>yes</Assertion>

<Type>united-states-citizen</Type>

$</$ VoterClassification>

$<$ VoterClassification $>$

$<$ Assertion>yes</Assertion>

$<$ otherType>ohio-resident</0therType $>$

$<$ Type >other</Type>

$</$ VoterClassification $>$

$<$ VoterClassification $>$

<Assertion>yes</Assertion>

<0therType>bmv-authorization</0therType>

$<$ Type>other</Type>

$</$ VoterClassification $>$

$<$ VoterId $>$

$<$ AttestNoSuchId $>$ false</AttestNoSuchId $>$

<StringValue $>A B 879456</$ StringValue $>$

$</$ VoterId $>$

$<$ Type $>$ drivers-license</Type $>$

$\langle$ VoterId $>$

$<$ AttestNoSuchId $>$ true $</$ AttestNoSuchId $>$

$<$ Type $>$ ssn4</Type $>$

$</$ VoterId $>$

$</$ Subject $>$

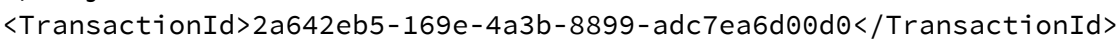

<Type>registration</Type>

$<$ VendorApplicationId $>$ OLVR $2.0</$ VendorApplicationId $>$

</VoterRecordsRequest> 


\subsection{Example 2: NVRA-style Voter Registration Request in JSON}

Figure 60 shows a fictitious NVRA-style voter registration request for Jane A. Doe in the State of Ohio using JSON.

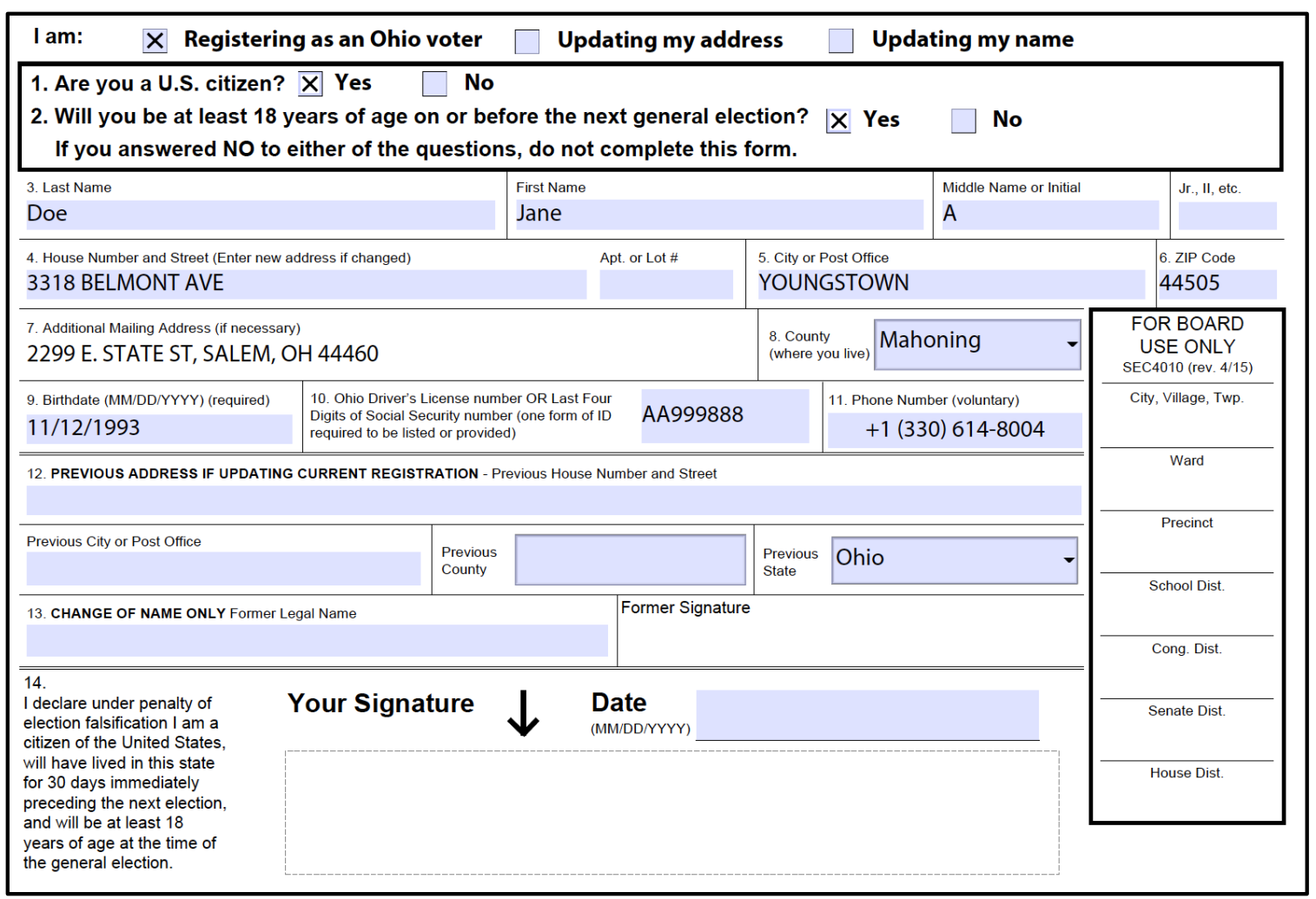

Figure 60 - Example NVRA-style form for a voter registration request

An example of the JSON statements for the voter registration request is shown below. Again, "AdditionalInfo" is being used in lines 3 through 14 to add information for elements not included in the JSON (and XML) schema, namely for the voter's preferred language and whether the voter wishes to volunteer as a poll worker. 


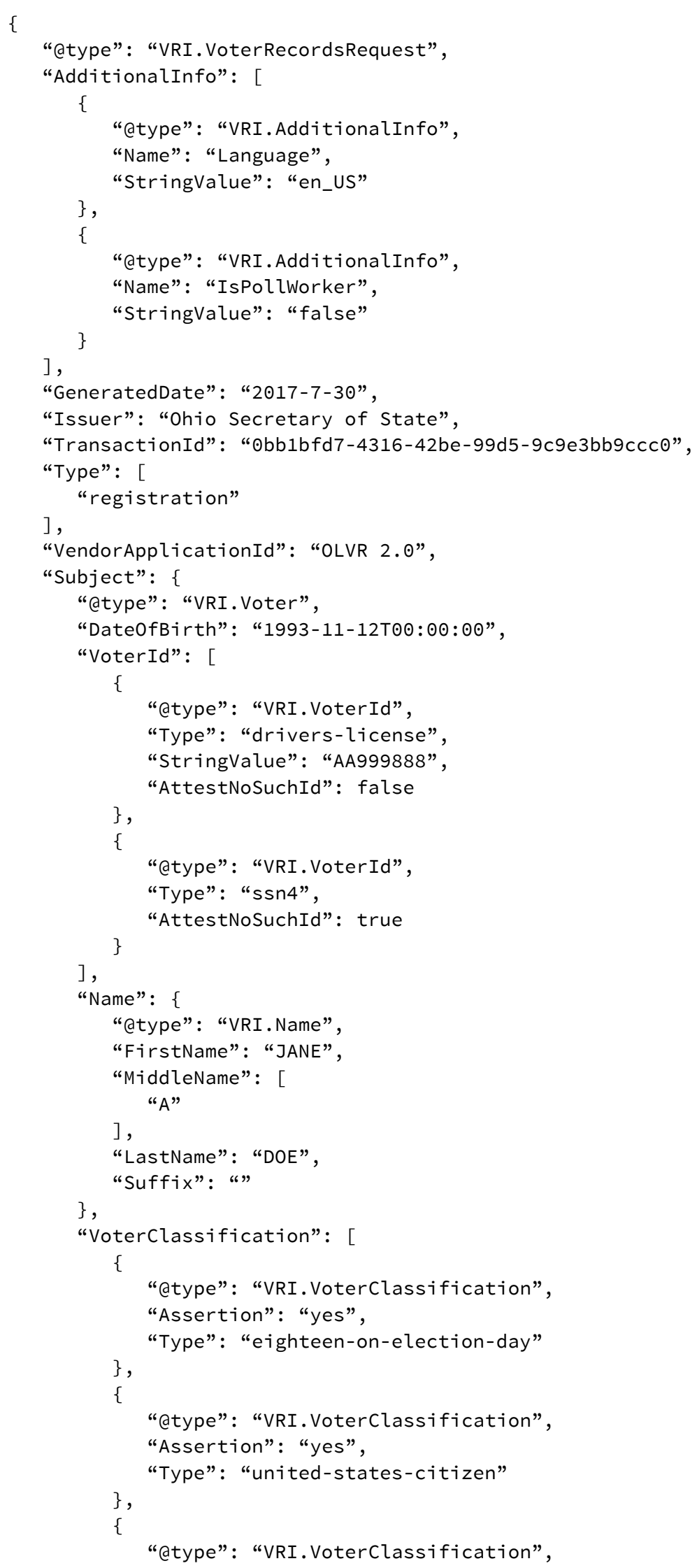


"Assertion": "yes",

"Type": "other",

"OtherType": "swear-accuracy"

\},

\{

"atype": "VRI.VoterClassification",

"Assertion": "yes",

"Type": "other",

"OtherType": "filled-on-own-behalf"

\},

\{

"atype": "VRI.VoterClassification",

"Assertion": "yes",

"Type": "other",

"OtherType": "ohio-resident"

\},

\{

"@type": "VRI.VoterClassification",

"Assertion": "yes",

"Type": "other",

"OtherType": "bmv-authorization"

\},

\{

"atype": "VRI.VoterClassification",

"Assertion": "yes",

"Type": "other",

"OtherType": "meets-all-requirements"

\}

],

"ContactMethod": [

\{

"atype": "VRI.ContactMethod",

"Type": "phone",

"Value": "(330) 614-8004"

\},

\{

"Atype": "VRI.ContactMethod",

"Type": "email",

"Value": "JDOE@TESTEMAIL.COM"

98

99

100

101

102

103

104

105

106

107

108

109

110

111

112

113

114

115

116

117

118

\}

],

"ResidenceAddress": \{

"atype": "addr.NumberedThoroughfareAddress_type",

"CompleteAddressNumber": \{

"@type": "addr_type.CompleteAddressNumber_type",

"AddressNumber": "3818"

\},

"CompleteStreetName": \{

"@type": "addr_type.CompleteStreetName_type",

"StreetNamePreDirectional": \{

"atype": "addr_type.StreetNamePreDirectional_type",

"Value": “"

\},

"StreetName": "BELMONT",

"StreetNamePostType": \{

"@type": "addr_type.StreetNamePreType_type",

"Value": "AVE"

\},

"StreetNamePostDirectional": \{ 
119

120

121

122

123

124

125

126

127

128

129

130

131

132

133

134

135

136

137

138

139

140

141

142

143

144

145

146

147

148

149

150

151

152

153

154

155

156

157

158

159

160

161

162

163

164

165

166

167

168

169

170

171

172

173

174

175

176

177

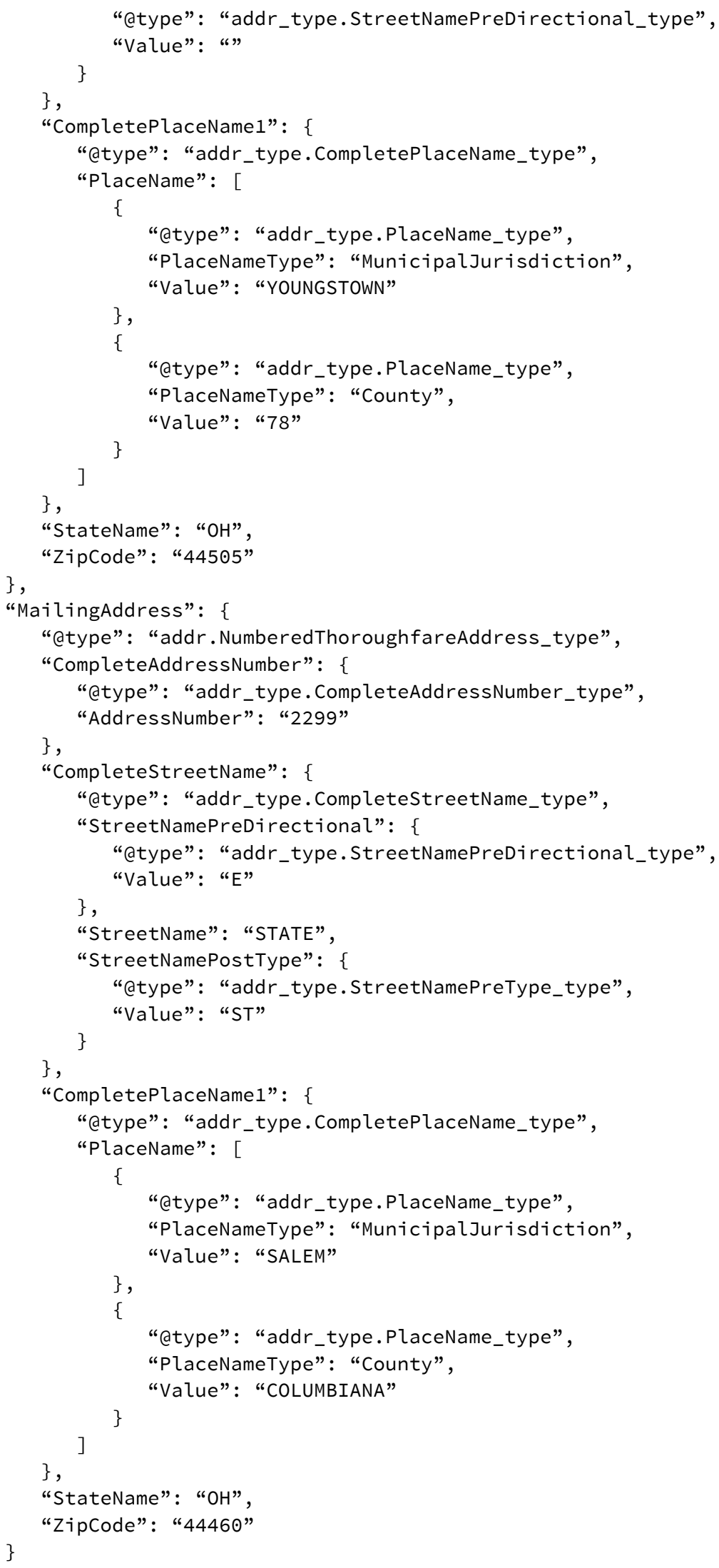


178

179

180

181

"RequestMethod": "voter-via-internet", "Form": "other",

"OtherForm": "4010" 


\subsection{Example 3: NVRA-style Voter Records Response in XML}

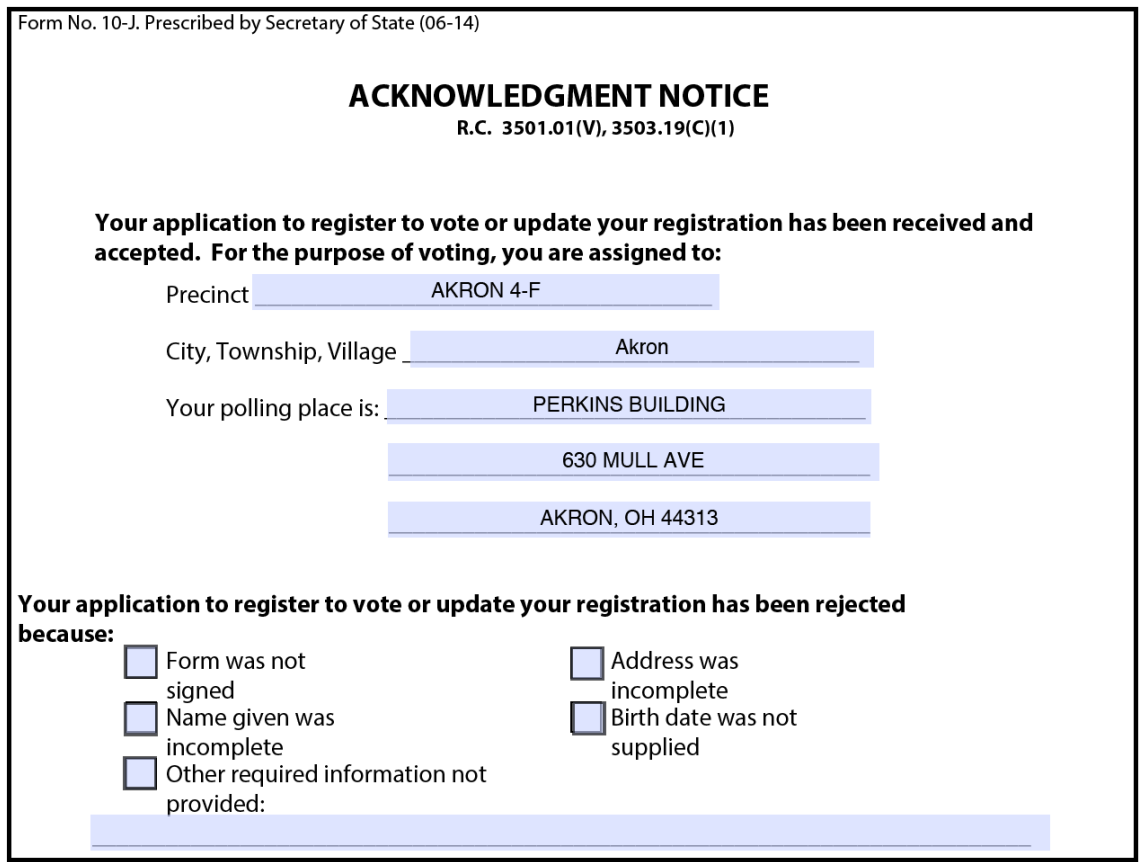

Figure 61 - Populated NVRA voter registration response form

This final example shows a fictitious digital NVRA-style registration response in the State of Ohio using XML. An example of the populated response form is shown in Figure 61. The XML for the voter registration response that contains the information used to populate the form is as follows: 


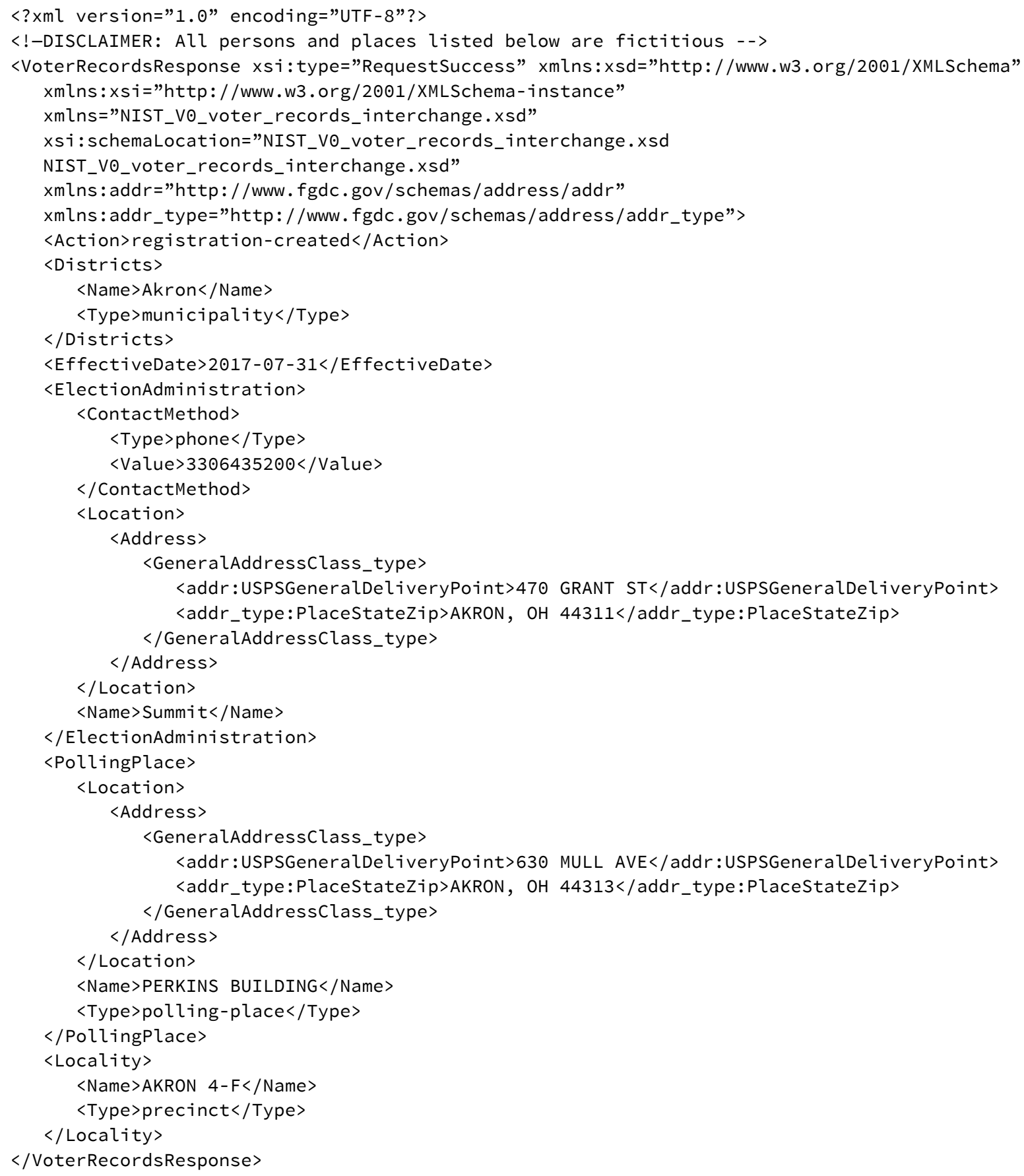




\section{Appendix A-Acronyms}

Selected acronyms and abbreviations used in this document are defined below.

\begin{tabular}{|c|c|}
\hline $\mathrm{CDF}$ & Common Data Format \\
\hline DMV & Department of Motor Vehicles \\
\hline EAC & Election Assistance Commission \\
\hline EAVS & EAC Election Administration and Voting Survey \\
\hline FIPS & Federal Information Processing Standard \\
\hline FPCA & Federal Post Card Application \\
\hline FWAB & Federal Write-in Absentee Ballot \\
\hline JSON & JavaScript Object Notation \\
\hline MMS & Multimedia Messaging Service \\
\hline MIME & Multipurpose Internet Mail Extensions \\
\hline MVA & Motor Vehicles Administration \\
\hline NIST & National Institute of Standards and Technology \\
\hline NVRA & National Voter Registration Act \\
\hline OCD-ID & Open Civic Data Identifiers \\
\hline OVR & Online Voter Registration \\
\hline SMS & Short Message Service \\
\hline SP & Special Publication \\
\hline UML & Unified Modeling Language \\
\hline UOCAVA & Uniform and Overseas Citizens Assistance in Voting Act \\
\hline VR & Voter Registration \\
\hline VRI & Voter Records Interchange \\
\hline VVSG & Voluntary Voting Systems Guidelines \\
\hline
\end{tabular}


XML

eXtensible Markup Language 


\section{Appendix B-Glossary}

Selected terms used throughout this document are defined below.

Election district:

Election official:

Polling place:

Precinct:

Registration assistant:

Registration proxy:

Registration witness:

Reporting unit:

Schema:
Administrative area in which voters are entitled to vote in contests that are specific to that area.

Any person who is involved with administering or conducting an election, including government personnel and temporary election workers. This may include any county clerk and recorder, election judge, member of a canvassing board, central election official, election day worker, member of a board of county commissioners, member or secretary of a board of directors authorized to conduct public elections, representative of a governing body, or other person engaged in the performance of election duties as required by the election code.

Location at which voters may cast in-person ballots under the supervision of election workers during one or more specific time periods.

Election administration division corresponding to a geographic area that is the basis for determining which contests the voters legally residing in that area are eligible to vote on.

An organization whose purpose includes assisting voters in registering to vote.

An organization that submits a voter registration request on behalf of the voter, e.g., a MVA office that submits a voter registration request for a voter.

An individual who witnesses a voter's registration, i.e., the voter signing his/her registration form.

Geographical area in which reported totals or counts are reported (for example, a jurisdiction, precinct, or election district).

A file containing definitions of data elements and attributes with rules for usage, e.g., for XML. 
UOCAVA voter:

An overseas voter or an active duty member of the U.S. military, either within or outside the United States, including any accompanying spouse and family members who are eligible to vote in their last place of residence in the United States. The Uniformed and Overseas Citizens Absentee Voting Act is commonly referred to as UOCAVA. 


\section{Appendix C-References}

[1]

[2]

[3]

[4]

[5]

[6]

[7]

W3C, Extensible Markup Language (XML) 1.0 (Fifth Edition), W3C

Recommendation, November 26, 2008, http://www.w3.org/TR/xml/ [accessed 2/5/2019].

JavaScript Object Notation, http://www.ecmainternational.org/publications/files/ECMA-ST/ECMA-404.pdf [accessed 2/5/2019].

The National Voter Registration Act of 1993, https://www.justice.gov/crt/national-voter-registration-act-1993-nvra [accessed 2/5/2019].

Federal Voting Assistance Program (FVAP), Federal Post Card Application (FPCA), https://www.fvap.gov/eo/overview/materials/forms [accessed 2/5/2019].

Object Management Group (OMG), UML Specification version 1.1 (OMG document ad/97-08-11) September 22, 2011, http://omg.org/ [accessed 2/5/2019].

Election Assistance Commission, Election Administration and Voting Survey, http://www.eac.gov/research/election_administration_and_voting_survey.aspx [accessed 2/5/2019].

Federal Geographic Data Committee (FGDC), United States Thoroughfare, Landmark, And Postal Address Data Standard, http://www.fgdc.gov/standards/projects/FGDC-standards-projects/addressdata/index html [accessed 2/5/2019].

Electronic Registration Information Center (ERIC), http://www.ericstates.org/ [accessed 2/5/2019].

Federal Geographic Data Committee (FGDC), https://www.fgdc.gov/ [accessed 2/5/2019].

U.S. Postal Service (USPS), "Postal Addressing Standards" Publication 28, April 2010, http://pe.usps.gov/cpim/ftp/pubs/Pub28/Pub28.pdf [accessed 2/5/2019].

Open Civic Data, OCD Identifiers, http://opencivicdata.readthedocs.org/en/latest/ocdids.html [accessed 2/5/2019]. 
[12] ISO 3166-1 Country Codes, https://www.iso.org/obp/ui/\#search [accessed 2/5/2019]. 


\section{Appendix D-File Download Locations}

The files associated with this specification are available for download from a NIST repository, whose address is:

https://github.com/usnistgov/VoterRecordsInterchange

These files are also available from:

http://vote.nist.gov

The files include:

- This specification,

- UML model,

- XML and JSON schemas, and

- Example files. 


\section{Appendix E-Change Log}

\section{Version 1 Release 2 - March 31, 2020}

- In the UML documentation, corrected the order of UML class attributes and enumeration values in the tables so that they match the order in the UML model and in the JSON and XML schemas.

- In the UML documentation, mentions of class and attribute names are properly linked to each other. Also done for mentions of enumeration and value names. 